The Astrophysical Journal, 687:566-578, 2008 November 1

(C) 2008. The American Astronomical Society. All rights reserved. Printed in U.S.A.

\title{
COMMON PROPER MOTION COMPANIONS TO NEARBY STARS: AGES AND EVOLUTION
}

\author{
V. V. MaKarov \\ Michelson Science Center, Mail Stop 100-22, California Institute of Technology, 770 South Wilson Avenue, Pasadena, CA 91125
}

AND

N. Zacharias And G. S. Hennessy

US Naval Observatory, 3450 Massachusetts Avenue, NW, Washington, DC 20392-5420; vvm@caltech.edu Received 2008 March 13; accepted 2008 July 1

\begin{abstract}
A set of 41 nearby stars (closer than $25 \mathrm{pc}$ ) is investigated which have very wide binary and common proper motion (CPM) companions at projected separations between 1000 and 200,000 AU. These companions are identified by astrometric positions and proper motions from the NOMAD catalog. Based mainly on measures of chromospheric and X-ray activity, age estimation is obtained for most of 85 identified companions. Color-absolute magnitude diagrams are constructed to test whether CPM companions are physically related to the primary nearby stars and have the same age. Our carefully selected sample includes three remote white dwarf companions to main-sequence stars and two systems (55 Cnc and GJ 777A) of multiple planets and distant stellar companions. Ten new CPM companions, including three of extreme separations, are found. Multiple hierarchical systems are abundant; more than $25 \%$ of CPM components are spectroscopic or astrometric binaries or multiples themselves. Two new astrometric binaries are discovered among nearby CPM companions, GJ 264 and HIP 59000, and preliminary orbital solutions are presented. The Hyades kinematic group (or stream) is presented broadly in the sample, but we find few possible thick-disk objects and no halo stars. It follows from our investigation that moderately young (age $\lesssim 1 \mathrm{Gyr}$ ) thin-disk dwarfs are the dominating species in the near CPM systems, in general agreement with the premises of the dynamical survival paradigm.
\end{abstract}

Subject headings: binaries: general — stars: kinematics

\section{INTRODUCTION}

Components of wide stellar binaries and common proper motion pairs have been drawing considerable interest for many years. Despite the increasing accuracy of observations and the growing range of accessible wavelengths, the origin of very wide, weakly bound, or unbound systems remains an open issue. Lépine \& Bongiorno (2007) estimated that at least $9.5 \%$ of stars within $100 \mathrm{pc}$ have companions with projected separations greater than $1000 \mathrm{AU}$. The renewed interest was boosted by the detection of a dearth of substellar mass companions in spectroscopic binaries and by the attempts to account for the missing late-type members of the near solar neighborhood.

The main objective of this paper is to investigate a well-defined set of nearby stars in very wide CPM pairs and to discover new pairs, possibly with low-mass companions. The secondary goal of our investigation is to establish or estimate the age and evolutionary status of bona fide companions using a wide range of available astrometric and astrophysical data. The origin and status of wide CPM systems is still a mystery, because most of them are likely unbound or very weakly bound and are expected to be easily disrupted in dynamical interactions with other stars or molecular clouds $(\S 3)$. The nearest stars to the Sun, $\alpha$ Cen and Proxima Cen, form a wide pair which may be on a hyperbolic orbit (Anosova \& Orlov 1991). It is expected that such systems should be mostly young, or belong to moving groups, remnant clusters or associations, but this has not yet been demonstrated on a representative sample. It is not known if the companions formed together and have the same age. We combine age-related parameters and data, including color-absolute magnitude diagrams $(\S 4)$, chromospheric activity indices $(\S 6.1)$, coronal X-ray luminosity $(\S 6.2)$, multiplicity parameters $(\S 7)$, and kinematics $(\S 8)$ to shed light on this problem.
Previous investigations in the field are too numerous to be listed, but a few papers in considerable overlap with this study should be mentioned. Poveda et al. (1994) published a catalog of 305 nearby wide binary and 29 multiple systems. They discussed the importance of moving groups for separating different species of wide binaries and tentatively assigned 32 systems to the Hyades stream (called a supercluster, following Eggen's nomenclature) and 14 to the Sirius stream. Salim \& Gould (2003) undertook a comprehensive revision of the Luyten catalog for approximately $44 \%$ of the sky, drastically improving precision of epoch 2000 positions and proper motions, and supplying the stars with NIR magnitudes from 2MASS. This allowed Gould \& Chaname (2004) to estimate, for the first time, trigonometric parallaxes of 424 common proper motion companions to Hipparcos stars with reliable parallaxes. This extrapolation of parallaxes to CPM companions is justified for high proper motion pairs where the rate of chance alignments is small. There is significant overlap between the sample investigated in this paper and the catalog of Gould \& Chaname (2004), although we did not use the latter as a starting point for our selection. We are also employing the parallax extrapolation technique for dim companions not observed by Hipparcos when constructing color-magnitude diagrams in this paper.

\section{SELECTING CPM SYSTEMS}

Our selection of candidate CPM systems was based on the Naval Observatory Merged Astrometric Dataset (NOMAD; Zacharias et al. 2004b), ${ }^{1}$ which provides an all-sky catalog of astrometric and photometric data. NOMAD includes astrometric data from the UNSO-B (Monet et al. 2003), UCAC2 (Zacharias et al. 2004a),

\footnotetext{
${ }^{1}$ At http://www.nofs.navy.mil/nomad.
} 
Hipparcos (ESA 1997), Tycho-2 (Høg et al. 2000) catalogs, and the Yellow Sky data set (D. Monet, private communication), supplemented by $B V R$ optical photometry, mainly from USNO-B, and $J H K$ near-IR photometry from $2 \mathrm{MASS}$. This catalog covers the entire magnitude range from the brightest naked eye stars to the limit of the POSS survey plates (about 21 st magnitude). The largest systematic positional errors are estimated for the Schmidt plate data used in the USNO-B catalog, with possible local offsets up to about 300 mas. Systematic errors in UCAC2 and 2MASS are much smaller (Zacharias et al. 2006). Thus, possible systematic errors of proper motions in NOMAD for the entries taken from

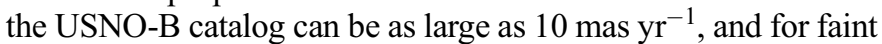
UCAC2 stars up to about $5 \mathrm{mas} \mathrm{yr}^{-1}$. Internal random errors of proper motions are given for all stars in the NOMAD catalog;

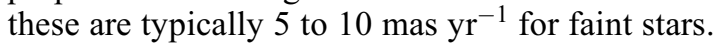

We used the following four criteria to select candidate CPM systems for this investigation: (1) at least one of the components should be listed in both Hipparcos and NOMAD; (2) the Hipparcos parallax of the primary component should be statistically reliable and greater than 40 mas (distance less than $25 \mathrm{pc}$ ); (3) at least one companion to the primary is found in NOMAD within $1.5^{\circ}$ at epoch $\mathrm{J} 2000.0$, whose proper motion is within a tolerance limit of the primary's proper motion; (4) the companion should be clearly visible in both DSS and 2MASS surveys, and be listed in 2MASS with $J, H$, and $K_{s}$ magnitudes. Some 1200 stars from the Hipparcos Catalogue with a parallax of 40 mas or larger were selected as initial targets. The tolerance limit was set at 8 mas $\mathrm{yr}^{-1}$ if the difference of the primary's Hipparcos and Tycho-2 proper motions was larger than this value in at least one of the coordinate components, and at 15 mas $\mathrm{yr}^{-1}$ otherwise. In addition, the difference in proper motion between the primary and the candidate companion was required to be within the $3 \sigma$ formal error on the NOMAD proper motion. These fairly strict limits removed from our analysis some known or suspected nearby pairs, for example, the nearest stars Alpha and Proxima Cen, which differ in proper motion by more than a hundred milliarcseconds per year. The resulting list of candidates was inspected by eye to exclude numerous erroneous NOMAD entries. In this paper we consider only CPM systems with projected separations greater than $1000 \mathrm{AU}$.

Table 1 lists 41 CPM systems, including 2 resolved triple systems. Alternative names are given for all companions, giving preference to Hipparcos numbers, various Luyten designations, and Gliese-Jahreiss identifications. The sources of J2000.0 ICRS positions are Hipparcos and NOMAD. The VI photometry comes mostly from (Bessel 1990; Weis 1991, 1993, 1996; Koen et al. 2002; Reid et al. 2002; Roselló et al. 1987) and for several stars from our own observations.

\section{DYNAMICAL SURVIVAL AND ORIGINS}

Very wide stellar systems of low binding energy encounter other stars and molecular clouds as they travel in the Galaxy, and these dynamical interactions are the main cause for stochastic evolution of their orbits and, in most cases, eventual disruption. Analytical considerations of dynamical evolution and survival of wide systems is limited to two asymptotic approximations, those of very distant and weak (but frequent) interaction, and "catastrophic" encounters at small impact parameters, which are rare but can be disruptive. We discuss in this paper the second kind of interactions. According to Weinberg et al. (1987) catastrophic encounters are defined as those with impact parameters $b<b_{\mathrm{BF}}$, where $b_{\mathrm{BF}}$ is defined, in analogy with Fokker-Planck diffusion coefficients, as the critical impact parameter at which the expected var- iance of total energy is equal to a certain fraction of the total energy squared:

$$
\sigma_{\Delta E}^{2}=\epsilon E^{2}
$$

The following proportionality relations are derived from (Weinberg et al. 1987) for the rates of catastrophic encounters:

$$
\begin{aligned}
& \Gamma_{\text {cat }} \propto n a \epsilon^{-1}\left\langle\frac{1}{V_{\text {rel }}}\right\rangle\left(b_{\mathrm{BF}} \ll a\right), \\
& \Gamma_{\text {cat }} \propto n a 32 \epsilon^{-(1 / 2)}\left(b_{\mathrm{BF}} \gg a\right),
\end{aligned}
$$

where $n$ is the perturber number density, $a$ is the semimajor axis of the binary system, $\left\langle 1 / V_{\text {rel }}\right\rangle$ is the mean reciprocal relative velocity of encounters.

The first limiting case, $b_{\mathrm{BF}} \ll a$, corresponds to encounters with individual stars, while the second, $b_{\mathrm{BF}} \gg a$, is a suitable approximation for encounters with dense cores of molecular clouds. Note that in the latter case, the rate of high-energy interactions is independent of the relative velocity.

The rate of disruptive interactions for both scenarios is proportional to the number density of perturbers $n$. It becomes immediately clear that the rate of disruption of very wide binaries is drastically different for the three major dynamical constituents of the Galaxy, the thin disk, the thick disk, and the halo.

The halo stars populating the outer, spherical component of the Galaxy have by far the largest velocities when they happen to travel in our neighborhood. The mean velocity with respect to the local standard of rest is directly related to the dispersions of velocity components $\left(\sigma_{U}, \sigma_{V}, \sigma_{W}\right)$. The "pure" halo, according to Chiba \& Beers (2000), is characterized by a prograde rotation of $V_{\phi} \simeq 30$ to $50 \mathrm{~km} \mathrm{~s}^{-1}$ and a dispersion ellipsoid of $\left(\sigma_{U}, \sigma_{V}\right.$, $\left.\sigma_{W}\right)=(141 \pm 11,106 \pm 9,94 \pm 8) \mathrm{km} \mathrm{s}^{-1}$. The vertical velocity component has immediate dynamical implications for wide binaries. The number density of molecular clouds, as well as of field stars is nonuniform in the vertical dimension, with a cusp at $z=0$. Wide binaries from the halo cross the densest part of the disk very quickly and spend most of their time hovering far from the plane where the density of perturbers is much lower. On the contrary, the thin-disk stars spend most of the time within the densest parts of the Galaxy, oscillating with small amplitudes around its midplane. These dynamical differences has dramatic implications for the typical survival time of very wide binaries. We can quantify the differences in the following way.

According to the numerical simulations of galactic motion in Makarov et al. (2004), the vertical oscillation is harmonic to firstorder approximation, with a period

$$
P_{\nu}\left(v_{z 0}\right) \simeq P_{\nu, H}\left[1+\frac{\left|v_{z 0}\right|}{10^{4}}\left(1.45+3.29\left|v_{z 0}\right|\right)\right],
$$

where $v_{z 0}$ is midplane vertical velocity in kilometers per second and $P_{\nu, H}=77.7 \mathrm{Myr}$ is the asymptotic harmonic period at $v_{z 0} \rightarrow 0$. This equation holds within $\pm 0.5 \%$ for $0 \leqslant v_{z 0} \leqslant 21 \mathrm{~km} \mathrm{~s}^{-1}$. Another useful equation relates the maximum excursion from the $\mathrm{Ga}-$ lactic plane with the midplane velocity:

$$
z_{\max }=12.044\left|v_{z 0}\right|
$$

Assuming typical midplane velocities to be equal to vertical dispersion estimates from Torra et al. (2000) for young stars, Famaey 
TABLE 1

Examined CPM Double and Multiple Systems within 25 pc

\begin{tabular}{|c|c|c|c|c|c|c|c|c|c|c|c|c|}
\hline $428 \ldots \ldots \ldots \ldots \ldots \ldots \ldots \ldots$ & GJ 2 & $\begin{array}{lll}00 & 05 & 10.8882\end{array}$ & +454711.641 & 328.1 & 870 & -151 & $86.98[1.41]$ & 9.97 & 2.53 & 6.70 & 6.10 & 5.85 \\
\hline $4849 \ldots \ldots \ldots \ldots \ldots \ldots \ldots .$. & GJ $3071 \mathrm{AB}$ & 010224.5721 & +050341.209 & & 340 & 221 & $46.61[1.61]$ & 8.15 & & 6.20 & 5.68 & 5.51 \\
\hline 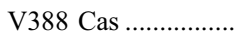 & GJ 51 & 010319.8653 & +622155.930 & 294.7 & 732 & 80 & & 13.78 & 3.32 & 8.61 & 8.01 & 7.72 \\
\hline 5799 & GJ $9045 \mathrm{~A}$ & 011424.0398 & -075522.173 & & 124 & 278 & $41.01[0.89]$ & 5.14 & 0.54 & 4.40 & 4.02 & 4.06 \\
\hline LTT $683 .$. & GJ 9045 B & 011422.4332 & -075439.232 & 49.1 & 123 & 272 & & 7.83 & 0.83 & 6.40 & 6.02 & 5.88 \\
\hline 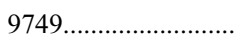 & GJ $9070 \mathrm{~A}$ & 020523.6559 & -280411.032 & & 340 & 422 & $44.37[1.97]$ & 10.96 & 1.69 & 7.99 & 7.35 & 7.16 \\
\hline $14555 \ldots \ldots \ldots \ldots \ldots \ldots$ & GJ $1054 \mathrm{~A}$ & 030755.7489 & -281311.013 & & -339 & -120 & $55.5[2.5]$ & 10.24 & 1.70 & 7.24 & 6.58 & 6.37 \\
\hline LTT $1477 \ldots \ldots \ldots \ldots \ldots . . .$. & GJ $1054 \mathrm{~B}$ & 030753.3793 & -281409.650 & 66.5 & -336 & -112 & & 13.09 & 2.31 & 9.35 & 8.78 & 8.52 \\
\hline $15330 \ldots \ldots \ldots \ldots \ldots \ldots \ldots \ldots$ & GJ 136 & 031746.1635 & -623431.160 & & 1338 & 649 & $82.51[0.54]$ & 5.53 & 0.71 & 4.46 & 4.04 & 3.99 \\
\hline $15371 \ldots \ldots \ldots \ldots \ldots \ldots \ldots \ldots$ & GJ 138 & $\begin{array}{llll}03 & 18 & 12.8189\end{array}$ & -623022.907 & 309.2 & 1331 & 647 & $82.79[0.53]$ & 5.24 & 0.68 & 4.27 & 3.87 & 3.86 \\
\hline $17414 \ldots \ldots$ & GJ $9122 \mathrm{~A}$ & 034352.5624 & +164019.272 & & 155 & -320 & $58.09[1.98]$ & 9.96 & 1.65 & 7.05 & 6.41 & 6.25 \\
\hline $17405 \ldots \ldots \ldots \ldots \ldots \ldots$ & GJ 9122 B & 034345.2490 & +164002.138 & 106.5 & 159 & -313 & $61.40[2.37]$ & 10.81 & 1.94 & 7.53 & 6.91 & 6.69 \\
\hline $21482 \ldots \ldots \ldots \ldots \ldots \ldots \ldots$ & V833 Tau & 043648.2425 & +270755.897 & & 232 & -147 & $56.02[1.21]$ & 8.10 & 1.60 & 5.95 & 5.40 & 5.24 \\
\hline WD $0433+270 \ldots \ldots$. & NLTT 13599 & 043644.8902 & +270951.594 & 124.0 & 226 & -153 & & 15.81 & 0.80 & 14.60 & 14.23 & 14.14 \\
\hline $22498 \ldots \ldots \ldots \ldots \ldots \ldots \ldots$ & DP Cam & 045025.0911 & +631958.624 & & 219 & -195 & $42.59[17.78]$ & 9.83 & 1.22 & 7.55 & 6.95 & 6.80 \\
\hline \multirow[t]{2}{*}{$42748 \ldots \ldots \ldots \ldots \ldots \ldots \ldots \ldots$} & GJ 319 A & 084244.5315 & +093324.114 & & 216 & -634 & $74.95[13.82]$ & 9.63 & 1.86 & 6.69 & 6.05 & 5.83 \\
\hline & GJ 319 C & 084252.2287 & +093311.157 & 114.6 & 224 & -616 & & 11.81 & 2.39 & 8.12 & 7.49 & 7.28 \\
\hline 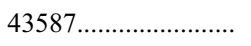 & GJ 324 A & 085235.8111 & +281950.947 & & -485 & -234 & $79.80[0.84]$ & 5.96 & & 4.77 & 4.26 & 4.01 \\
\hline LTT $12311 \ldots \ldots \ldots \ldots . . .$. & GJ 324 B & 085240.8393 & +281859.310 & 84.1 & -488 & -234 & & 13.14 & 3.00 & 8.56 & 7.93 & 7.67 \\
\hline $46843 \ldots \ldots \ldots \ldots \ldots \ldots \ldots$ & GJ $9301 \mathrm{~A}$ & 093243.7592 & +265918.708 & & -148 & -246 & $56.35[0.89]$ & 7.01 & & 5.58 & 5.24 & 5.12 \\
\hline NLTT $22015 \ldots \ldots \ldots \ldots . .$. & GJ 9301 B & 093248.2450 & +265943.864 & 65.0 & -142 & -243 & & & & 10.36 & 9.86 & 9.47 \\
\hline $47620 \ldots \ldots \ldots \ldots \ldots \ldots \ldots$ & GJ 360 & 94234.8429 & +700201.989 & & -671 & -269 & $85.14[3.18]$ & 10.58 & 2.20 & 6.92 & 6.33 & 6.08 \\
\hline $47650 \ldots \ldots \ldots \ldots \ldots \ldots \ldots$ & GJ 362 & 094251.7315 & +7002 21.892 & 88.8 & -669 & -264 & $86.69[2.24]$ & 11.24 & 2.41 & 7.33 & 6.73 & 6.47 \\
\hline \multirow[t]{2}{*}{ 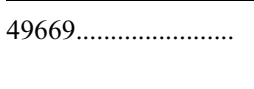 } & GJ $9316 \mathrm{~A}$ & 100822.3106 & +115801.945 & & -249 & 5 & $42.09[0.79]$ & 1.35 & & 1.67 & 1.66 & 1.64 \\
\hline & GJ 9316 B & 100812.7970 & +115949.078 & 176.0 & -244 & 12 & & 8.11 & 1.00 & 6.42 & 5.99 & 5.88 \\
\hline $50564 \ldots \ldots \ldots \ldots \ldots \ldots \ldots$ & GJ 9324 & 101944.1679 & +192815.290 & & -230 & -215 & $47.24[0.82]$ & 4.80 & & 4.04 & 3.94 & 4.02 \\
\hline NLTT $23781 \ldots \ldots \ldots . . .$. & & 101453.8493 & +202214.590 & 5220.6 & -232 & -212 & & 16.48 & & 10.81 & 10.20 & 9.99 \\
\hline $59000 \ldots \ldots \ldots \ldots \ldots \ldots$ & GJ 9387 & 120550.6574 & -185230.916 & & -19 & -320 & $44.41[1.51]$ & 9.95 & 1.57 & 7.42 & 6.79 & 6.62 \\
\hline NLTT $29580 \ldots \ldots \ldots . . .$. & & 120546.6407 & -184932.240 & 187.6 & -4 & -314 & & 16.23 & 3.32 & 11.20 & 10.63 & 10.32 \\
\hline 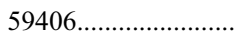 & GJ $3708 \mathrm{~A}$ & 121111.7583 & -195738.064 & & -216 & -184 & $78.14[2.80]$ & 11.68 & 2.33 & 7.89 & 7.36 & 7.04 \\
\hline NLTT 29879 ............. & GJ 3709 B & 121116.95 & -195821.9 & 85.2 & -203 & -188 & & 12.62 & 2.51 & 8.60 & 8.01 & 7.74 \\
\hline 61451............ & GJ $1161 \mathrm{~A}$ & & & & -228 & & $46.19[0.91]$ & 7.87 & & 5.95 & & 5.26 \\
\hline LTT $4788 \ldots \ldots \ldots \ldots \ldots . . .$. & GJ $1161 \mathrm{~B}$ & 123537.7821 & -345415.309 & 95.8 & -219 & -128 & & 11.76 & 2.39 & 8.15 & 7.58 & 7.30 \\
\hline $63882 \ldots \ldots \ldots \ldots \ldots \ldots$ & GJ 3760 & 130529.8783 & +370810.635 & & -304 & -202 & $43.18[6.95]$ & 10.62 & & 8.22 & 7.61 & 7.35 \\
\hline
\end{tabular}


TABLE 1 - Continued

\begin{tabular}{|c|c|c|c|c|c|c|c|c|c|c|c|c|}
\hline $\begin{array}{l}\mathrm{HIP} / \mathrm{Name} \\
\text { (1) }\end{array}$ & $\begin{array}{l}\text { Alt. Name } \\
\text { (2) }\end{array}$ & $\begin{array}{l}\text { R.A. } \\
\text { (J2000.0) } \\
\text { (3) }\end{array}$ & $\begin{array}{c}\text { Decl. } \\
\text { (J2000.0) } \\
(4)\end{array}$ & $\begin{array}{l}\text { Sep. } \\
(5)\end{array}$ & $\begin{array}{l}\mu_{\alpha} \cos \delta \\
(6)\end{array}$ & $\begin{array}{l}\mu_{\delta} \\
(7)\end{array}$ & $\begin{array}{c}\Pi\left[\sigma_{\Pi}\right] \\
\quad(8)\end{array}$ & $\begin{array}{c}V \\
(9)\end{array}$ & $\begin{array}{l}V-I \\
(10)\end{array}$ & $\begin{array}{c}J \\
(11)\end{array}$ & $\begin{array}{c}H \\
(12)\end{array}$ & $\begin{array}{c}K \\
(13)\end{array}$ \\
\hline 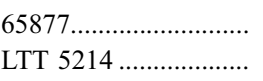 & GJ 515 & $\begin{array}{lll}13 & 30 & 13.6398 \\
13 & 30 & 02.8247\end{array}$ & $\begin{array}{lll}-08 & 34 & 29.492 \\
-08 & 42 & 25.530\end{array}$ & 502.3 & $\begin{array}{l}-1107 \\
-1102\end{array}$ & $\begin{array}{l}-475 \\
-472\end{array}$ & $55.50[3.77]$ & $\begin{array}{l}12.39 \\
14.33\end{array}$ & $\begin{array}{r}-0.01 \\
3.04\end{array}$ & $\begin{array}{r}12.62 \\
9.60\end{array}$ & $\begin{array}{r}12.68 \\
9.05\end{array}$ & $\begin{array}{r}12.74 \\
8.75\end{array}$ \\
\hline 71914 & $\begin{array}{l}\text { GJ } 9490 \text { A } \\
\text { LTT } 14350\end{array}$ & $\begin{array}{lll}14 & 42 & 33.6486 \\
14 & 42 & 26.2580\end{array}$ & $\begin{array}{l}+192847.219 \\
+193012.694\end{array}$ & 135.0 & $\begin{array}{l}-254 \\
-261\end{array}$ & $\begin{array}{l}-154 \\
-177\end{array}$ & $\begin{array}{l}44.54[2.57] \\
38.62[2.01]\end{array}$ & $\begin{array}{r}9.11^{\mathrm{a}} \\
10.08\end{array}$ & $\begin{array}{l}1.33 \\
1.43\end{array}$ & $\begin{array}{l}6.60 \\
7.45\end{array}$ & $\begin{array}{l}5.97 \\
6.80\end{array}$ & $\begin{array}{r}582 \\
\quad 6.66\end{array}$ \\
\hline 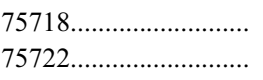 & $\begin{array}{l}\text { GJ } 586 \text { A } \\
\text { GJ } 586 \mathrm{~B}\end{array}$ & $\begin{array}{lll}15 & 28 & 09.6114 \\
15 & 28 & 12.2103\end{array}$ & $\begin{array}{l}-092053.050 \\
-092128.296\end{array}$ & 52.2 & $\begin{array}{l}73 \\
82\end{array}$ & $\begin{array}{l}-363 \\
-356\end{array}$ & $\begin{array}{l}50.34[1.11] \\
48.06[1.14]\end{array}$ & $\begin{array}{l}6.89 \\
7.54\end{array}$ & $\begin{array}{l}0.87 \\
0.91\end{array}$ & $\begin{array}{l}5.44 \\
5.99\end{array}$ & $\begin{array}{l}5.05 \\
5.55\end{array}$ & $\begin{array}{l}4.89 \\
5.46\end{array}$ \\
\hline 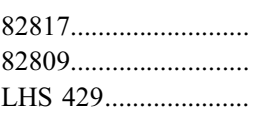 & $\begin{array}{l}\text { GJ } 644 \mathrm{AB} \\
\text { GJ } 643 \\
\text { GJ } 644 \mathrm{C}\end{array}$ & $\begin{array}{lll}16 & 55 & 28.7549 \\
16 & 55 & 25.2251 \\
16 & 55 & 35.2673\end{array}$ & $\begin{array}{lll}-08 & 20 & 10.838 \\
-08 & 19 & 21.274 \\
-08 & 23 & 40.840\end{array}$ & $\begin{array}{r}72.1 \\
231.2\end{array}$ & $\begin{array}{l}-829 \\
-813 \\
-810\end{array}$ & $\begin{array}{l}-879 \\
-895 \\
-872\end{array}$ & $\begin{array}{r}174.22[3.90] \\
153.96[4.04] \\
154.5[0.7]^{\mathrm{b}}\end{array}$ & $\begin{array}{c}9.02 \\
11.74 \\
16.85^{\mathrm{b}}\end{array}$ & $\begin{array}{l}2.33 \\
2.63 \\
4.54^{\mathrm{b}}\end{array}$ & $\begin{array}{l}5.27 \\
7.55 \\
9.78\end{array}$ & $\begin{array}{l}4.78 \\
7.06 \\
9.20\end{array}$ & $\begin{array}{l}4.40 \\
6.72 \\
8.82\end{array}$ \\
\hline 83599 & $\begin{array}{l}\text { GJ } 653 \\
\text { GJ } 654\end{array}$ & $\begin{array}{lll}17 & 05 & 13.7781 \\
17 & 05 & 03.3941\end{array}$ & $\begin{array}{lll}-05 & 05 & 39.220 \\
-05 & 03 & 59.428\end{array}$ & 184.5 & $\begin{array}{l}-921 \\
-917\end{array}$ & $\begin{array}{l}-1128 \\
-1138\end{array}$ & $\begin{array}{l}89.70[28.71] \\
92.98[1.04]\end{array}$ & $\begin{array}{r}10.09 \\
7.73\end{array}$ & & $\begin{array}{l}6.78 \\
5.52\end{array}$ & $\begin{array}{l}6.19 \\
4.94\end{array}$ & $\begin{array}{l}5.97 \\
4.73\end{array}$ \\
\hline 86036 & $\begin{array}{l}26 \text { Dra } \\
\text { GJ } 685\end{array}$ & $\begin{array}{l}173459.5940 \\
173534.4809\end{array}$ & $\begin{array}{l}+615228.394 \\
+614053.631\end{array}$ & 737.5 & $\begin{array}{l}277 \\
264\end{array}$ & $\begin{array}{l}-526 \\
-514\end{array}$ & $\begin{array}{l}70.98[0.55] \\
70.95[1.09]\end{array}$ & $\begin{array}{l}5.23 \\
9.97\end{array}$ & 1.81 & $\begin{array}{l}4.24 \\
6.88\end{array}$ & $\begin{array}{l}3.88 \\
6.27\end{array}$ & $\begin{array}{l}3.74 \\
6.07\end{array}$ \\
\hline 93899 & $\begin{array}{l}\text { GJ } 745 \mathrm{~B} \\
\text { GJ } 745 \mathrm{~A}\end{array}$ & $\begin{array}{lll}19 & 07 & 13.2039 \\
19 & 07 & 05.5632\end{array}$ & $\begin{array}{l}+205237.254 \\
+205316.973\end{array}$ & 114.2 & $\begin{array}{l}-481 \\
-481\end{array}$ & $\begin{array}{l}-333 \\
-346\end{array}$ & $\begin{array}{l}112.82[2.41] \\
115.91[2.47]\end{array}$ & $\begin{array}{l}10.76 \\
10.78\end{array}$ & $\begin{array}{l}2.09 \\
2.09\end{array}$ & $\begin{array}{l}7.28 \\
7.30\end{array}$ & $\begin{array}{l}6.75 \\
6.73\end{array}$ & $\begin{array}{l}6.52 \\
6.52\end{array}$ \\
\hline 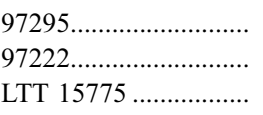 & $\begin{array}{l}\text { GJ } 9670 \mathrm{~A} \\
\text { LTT } 15766 \\
\text { GJ } 9670 \mathrm{~B}\end{array}$ & $\begin{array}{l}194625.6001 \\
194533.5520 \\
194627.5446\end{array}$ & $\begin{array}{l}+334339.351 \\
+333606.055 \\
+334348.894\end{array}$ & $\begin{array}{r}792.3 \\
25.8\end{array}$ & $\begin{array}{l}19 \\
23 \\
25\end{array}$ & $\begin{array}{l}-446 \\
-449 \\
-438\end{array}$ & $\begin{array}{l}47.94[0.54] \\
49.09[1.43]\end{array}$ & $\begin{array}{l}4.96 \\
7.68 \\
8.58\end{array}$ & 0.53 & $\begin{array}{l}4.05 \\
5.81 \\
6.64\end{array}$ & $\begin{array}{l}3.98 \\
5.32 \\
6.12\end{array}$ & $\begin{array}{l}3.83 \\
5.25 \\
6.00\end{array}$ \\
\hline $\begin{array}{l}102409 \ldots \ldots \ldots \ldots \ldots \ldots \ldots . . . \\
102141 \ldots \ldots \ldots \ldots \ldots \ldots . . .\end{array}$ & $\begin{array}{l}\text { GJ } 803 \\
\text { GJ } 799\end{array}$ & $\begin{array}{lll}20 & 45 & 09.5317 \\
20 & 41 & 51.1537\end{array}$ & $\begin{array}{lll}-31 & 20 & 27.238 \\
-32 & 26 & 06.730\end{array}$ & 4680.0 & $\begin{array}{l}261 \\
280\end{array}$ & $\begin{array}{l}-345 \\
-360\end{array}$ & $\begin{array}{r}100.59[1.35] \\
97.80[4.65]\end{array}$ & $\begin{array}{r}8.75 \\
10.33\end{array}$ & $\begin{array}{l}2.07 \\
2.92\end{array}$ & $\begin{array}{l}5.81 \\
5.44\end{array}$ & $\begin{array}{l}5.20 \\
4.83\end{array}$ & $\begin{array}{l}4.94 \\
4.53\end{array}$ \\
\hline 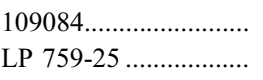 & GJ 4254 & $\begin{array}{lll}22 & 05 & 51.2986 \\
22 & 05 & 35.7280\end{array}$ & $\begin{array}{lll}-11 & 54 & 51.022 \\
-11 & 04 & 28.820\end{array}$ & 3030.9 & $\begin{array}{l}-266 \\
-274\end{array}$ & $\begin{array}{l}-175 \\
-162\end{array}$ & $46.70[7.86]$ & 10.15 & & $\begin{array}{r}7.22 \\
11.66\end{array}$ & $\begin{array}{r}6.60 \\
11.05\end{array}$ & $\begin{array}{r}6.40 \\
10.72\end{array}$ \\
\hline 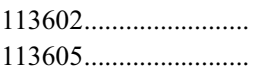 & $\begin{array}{l}\text { NLTT } 9310 \\
\text { NLTT } 9315\end{array}$ & $\begin{array}{lll}23 & 00 & 33.4015 \\
23 & 00 & 36.5922\end{array}$ & $\begin{array}{l}-235710.309 \\
-235810.657\end{array}$ & 74.5 & $\begin{array}{l}190 \\
195\end{array}$ & $\begin{array}{l}-345 \\
-346\end{array}$ & $\begin{array}{l}49.15[3.03] \\
49.36[3.19]\end{array}$ & $\begin{array}{l}11.57 \\
11.61\end{array}$ & $\begin{array}{l}1.95 \\
1.98\end{array}$ & $\begin{array}{l}8.25 \\
8.26\end{array}$ & $\begin{array}{l}7.67 \\
7.66\end{array}$ & $\begin{array}{l}7.41 \\
7.42\end{array}$ \\
\hline $\begin{array}{l}115147 \ldots \ldots \ldots \ldots \ldots \ldots \ldots \\
\text { LSPM J2322+7847 .... }\end{array}$ & V368 Cep & $\begin{array}{lll}23 & 19 & 26.6320 \\
23 & 22 & 53.8733\end{array}$ & $\begin{array}{l}+790012.666 \\
+784738.810\end{array}$ & 959.1 & $\begin{array}{l}201 \\
210\end{array}$ & $\begin{array}{l}72 \\
64\end{array}$ & $50.65[0.64]$ & $\begin{array}{r}7.54 \\
16.18\end{array}$ & 3.62 & $\begin{array}{r}5.90 \\
10.42\end{array}$ & $\begin{array}{l}5.51 \\
9.84\end{array}$ & $\begin{array}{l}5.40 \\
9.52\end{array}$ \\
\hline 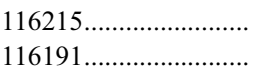 & $\begin{array}{l}\text { GJ } 898 \text { A } \\
\text { GJ } 897\end{array}$ & $\begin{array}{lll}23 & 32 & 49.3998 \\
23 & 32 & 46.5991\end{array}$ & $\begin{array}{l}-165044.308 \\
-164508.395\end{array}$ & 338.3 & $\begin{array}{l}344 \\
382\end{array}$ & $\begin{array}{l}-218 \\
-186\end{array}$ & $\begin{array}{c}71.70[1.36] \\
89.9[7.3]\end{array}$ & $\begin{array}{r}8.62 \\
10.43\end{array}$ & $\begin{array}{l}1.28 \\
2.24\end{array}$ & $\begin{array}{l}6.24 \\
6.71\end{array}$ & $\begin{array}{l}5.61 \\
6.09\end{array}$ & $\begin{array}{l}5.47 \\
5.86\end{array}$ \\
\hline
\end{tabular}

Notes.-Units of right ascension are hours, minutes, and seconds, and units of declination are degrees, arcminutes, and arcseconds. Col. (1): HIP number or name. Col. (2): Alternative name. Col. (3): Right ascension. Col. (4): Declination. Col. (5): Separation on the sky in arcseconds. Cols. (6) and (7): Proper motion in mas yr ${ }^{-1}$. Col. (8): Parallax and its error in mas. Col. (9): $V$ magnitude. Col. (10): $V-I$ color. Col. (11): $J$ magnitude. Col. (12): $H$ magnitude. Col. (13): $K$ magnitude.

${ }^{\text {a }}$ Our photometric observations.

b Photometry and parallax from Dahn et al. (2002).

et al. (2005) for thin-disk giants, and Chiba \& Beers (2000) for the thick disk and halo, we estimate characteristic midplane velocities, maximum vertical excursions, periods of oscillation, and the fraction of lifetime spend in the dense part of the Galaxy for these four dynamical components (Table 2). The latter parameter is de- fined as the fraction of an oscillation period when the star is within $100 \mathrm{pc}$ of the plane, $f(|z|<100)$.

The halo binaries cross the thin disk so quickly that their chances to encounter a perturber (a field star or a molecular core) are relatively slim. Thus, generic binaries of very low binding energy can 
TABLE 2

Vertical Motion of Galactic Components

\begin{tabular}{|c|c|c|c|c|}
\hline Component & $\begin{array}{c}\left|v_{z 0}\right| \\
\left(\mathrm{km} \mathrm{s}^{-1}\right)\end{array}$ & $\begin{array}{l}z_{\max } \\
(\mathrm{pc})\end{array}$ & $\begin{array}{c}P_{\nu} \\
(\mathrm{Myr})\end{array}$ & $f(|z|<100)$ \\
\hline Thin disk (young)............ & 6 & 72 & 79 & 1.00 \\
\hline Thin disk (giants) ............ & 18 & 217 & 86 & 0.31 \\
\hline Thick disk ........................... & 35 & 422 & 109 & 0.16 \\
\hline 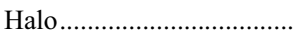 & 94 & 1130 & 305 & 0.07 \\
\hline
\end{tabular}

probably survive for a long time in the halo. However, these objects are rare in the solar neighborhood because of the intrinsic low number density, and none seem to be present in our sample. A typical thick disk binary may also stay intact much longer than a young disk binary, because it spends at least 6 times less time in the high-density midplane area. As far as encounters with stars are concerned, the difference in the time of survival can be even greater, because the average reciprocal velocity of encounter enters equation (2). Most of the interactions of thick-disk binaries with thin-disk perturbers will be rapid, further reducing the rate of disruptive events.

We can expect from this analysis that the distribution of very wide binaries and common proper motion pairs in age should be bimodal. Young CPM pairs in the thin disk, despite the higher rate of catastrophic interactions, can survive in significant numbers to this day. This kind of binary should be especially prominent if indeed most of the new stars are born in loose comoving groups such as the Lupus association of pre-main-sequence stars (Makarov 2007b).

\section{COLOR-ABSOLUTE MAGNITUDE DIAGRAMS}

Figure 1 represents the joint $M_{K s}$ versus $V-K_{s}$ color-absolute magnitude diagram for all resolved CPM companions listed in Table 1 that have $V$ and $J H K$ magnitudes. We assumed in constructing this diagram zero extinction for all stars, and we applied the Hipparcos parallaxes determined for primary stars to their CPM companions, unless the latter have independent trigonometric parallax measurements. Known unresolved binary or multiple stars are marked with inscribed crosses. A zero-age main sequence (ZAMS) and a $16 \mathrm{Myr}$ isochrone at solar metallicity $(Z=0.001$; from Siess et al. 2000) are drawn with thin lines, and the empirical main sequence from Henry et al. (2004) with a thick dashed line. Some of the interesting stars discussed later in this paper are labeled and named. Mutual positions of primary and secondary CPM companions are shown with dotted lines only for pairs with white dwarf companions.

The diagram shows that most of normal stars lie on or slightly above the main sequence. This confirms that the fainter CPM companions are probably physical. We find that many of the components lying close to the $16 \mathrm{Myr}$ isochrone (top solid line) are known visual, astrometric or spectroscopic binaries, which accounts for their excess brightness. For example, the primary component of the CPM pair HIP 66492 and NLTT 34706 is a resolved binary with a period $P=330 \mathrm{yr}$, semimajor axis $a=2.13^{\prime \prime}$, and eccentricity $e=0.611$ (Seymour et al. 2002). Formally, the joint magnitude can be as much as 0.75 brighter (in the case of twin companions) than the magnitude of the primary star. A number of components in Figure 1 lie significantly outside the upper envelope of unresolved binaries defined by the empirical main sequence minus $0.75 \mathrm{mag}$. Gross photometric errors (in particular, in $V$ for faint $\mathrm{M}$ dwarfs) cannot be completely precluded, but we suspect that most of these outlying stars should be either very young or unresolved multiple stars.

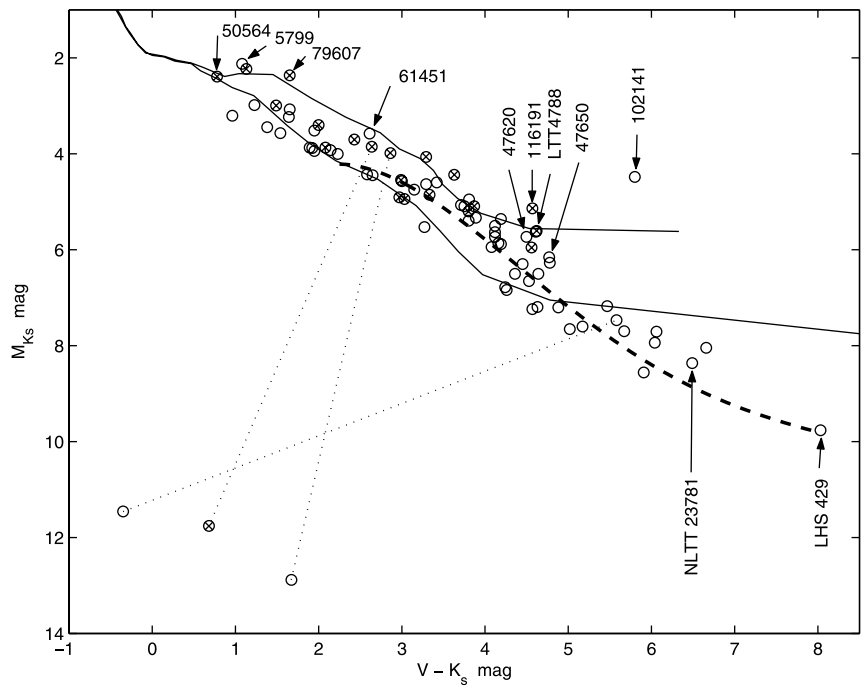

FIG. 1.-Joint color-absolute magnitude diagram of CPM pairs in $M_{K s}$ vs. $V-K_{s}$ axes. The ZAMS and $16 \mathrm{Myr}$ isochrones are drawn from (Siess et al. 2000 ), both for $Z=0.001$. The three white dwarfs of our sample are connected with their $\mathrm{M}$ dwarf companions by dotted straight lines. The thicker dashed line indicates the empirical main sequence for field dwarfs from Henry et al. (2004). Known unresolved binary companions of all kinds are marked with crosses inscribed in circles.

The CPM pair of HIP 61451 and LTT 4788 is an emphatic example of overluminous stars whose origin remains an unresolved issue. They match the $16 \mathrm{Myr}$ isochrone on the HR diagram very well. The $K_{s}$-band excess for these companions is 0.7 and $1.0 \mathrm{mag}$, respectively. In the literature we found no indication of binarity for either star. The primary component can still be binary with an almost twin companion, but the secondary should be at least triple to account for the near-infrared excess if it is a normal (old) M2.5 dwarf. On the basis of the kinematics of HIP 61451 and its excess luminosity, Eggen (1995) included it in his list of the Pleiades supercluster, which is synonymous with the Local Young Stream (Makarov \& Urban 2000). This may indicate an age between 1 and 125 Myr. Furthermore, HIP 61451 is a moderate and very soft X-ray emitter (Table 4), which may be expected of a post-T Tauri star. On the other hand, its level of chromospheric activity, at $\log R_{\mathrm{HK}}^{\prime}=-4.601$ (Gray et al. 2006), is not impressive, corresponding to an activity age of $\simeq 1 \mathrm{Gyr}($ see $\S 6)$. We propose that a careful investigation of the M-type CPM companion LTT 4788 should resolve the mystery of this system.

Figure 2 shows a color-absolute magnitude diagram of some selected CPM components discussed below in more detail, on $M_{V}$ versus $V-K_{S}$ axes. Each star is identified with its Hipparcos number or other name. The two thin lines show the ZAMS (bottom) and the $16 \mathrm{Myr}$ isochrone (top) from the models by Siess et al. (2000) both for $Z=0.001$ and zero extinction. The thicker dash-dotted line is the empirical main sequence for field stars from (Reid \& Cruz 2002). In this plot the CPM components are connected with thin dashed lines to show their relative position. Again, trigonometric parallaxes of the primary components were assumed for faint companions with unknown distances.

\section{NEW CPM PAIRS}

We report 10 new possible CPM companions and 8 new CPM systems, including 3 at extremely large separations, identified by our search procedure $(\S 2)$. Table 3 gives the Washington Double Star catalog (WDS; Mason et al. 2001) identifications for the primaries of known systems and indicates new systems and wide companions. The original discoverer references and other catalog 


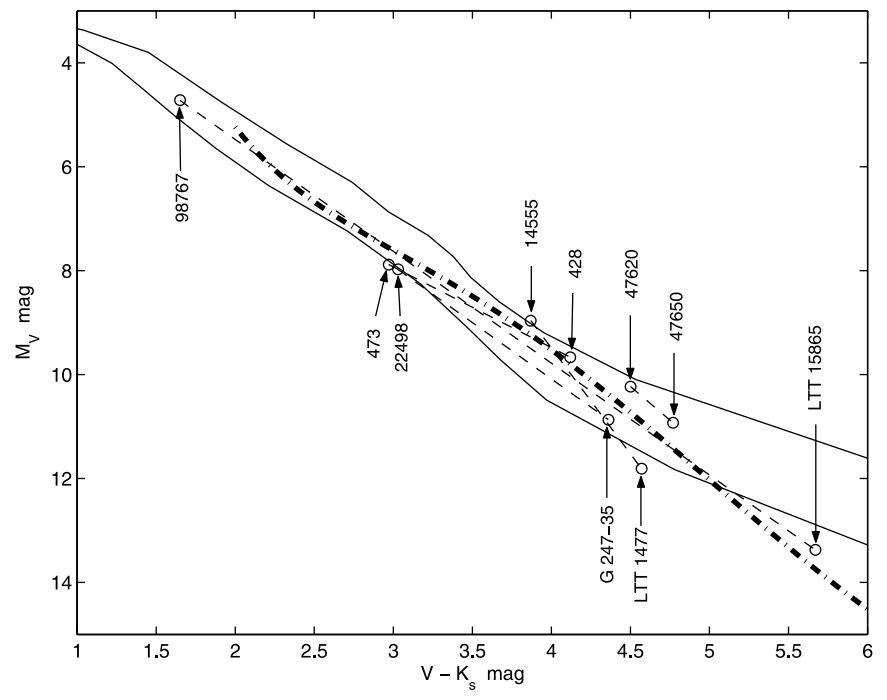

FIG. 2.-Color-absolute magnitude diagram of selected CPM companions in $M_{V}$ versus $V-K_{s}$ axes. The zero-age main sequence (ZAMS) and $16 \mathrm{Myr}$ isochrone are drawn from Siess et al. (2000), both for $Z=0.001$. Components of CPM pairs are connected by dashed straight lines. The thicker dot-dashed line indicates the empirical main sequence for field dwarfs from Reid \& Cruz (2002).

identifications can be found in WDS. In this section we discuss four probable new systems with peculiar characteristics, which may be interesting to pursue with additional photometric and spectroscopic observations.

HIP 109084 is a rather nondescript M0 dwarf at approximately $20 \mathrm{pc}$. This star has an uncertain parallax in the Hipparcos catalog with a formal error of 7.9 mas, most likely affected by unresolved binarity. According to Gizis et al. (2002), the $\mathrm{H} \alpha$ line is in absorption ( $\mathrm{EW}=-0.55 \AA$ ), hence the $\mathrm{H} \alpha$ lower limit on age is $150 \mathrm{Myr}(\S 6)$. Its alleged CPM companion LP 759-25, as one of the nearest and latest $\mathrm{M}$ dwarfs, has drawn more interest. PhanBao \& Bessell (2006) estimate a spectroscopic distance of $18 \mathrm{pc}$ for this star. At a projected separation of 65,000 AU, this may be one of the widest known CPM pairs, but more accurate astrometric information is required to verify the physical connection between these stars.

The K3 dwarf HIP 4849 at 21 pc from the Sun is a binary resolved by Hipparcos and with speckle interferometry (Fabricius \& Makarov 2000b; Balega et al. 2006). Its inner companion is probably a K8 dwarf orbiting the primary at $a=465$ mas with a period of $29 \mathrm{yr}$. We propose that this binary system has a distant CPM companion, the DA5 white dwarf WD 0101+048. The projected separation between the CPM components is 27,000 AU. The white dwarf companion is itself a binary star, having a spectroscopically detected close DC white dwarf companion (Maxted et al. 2000). The center-of-mass radial velocity of the WD pair is $63.4 \pm 0.2 \mathrm{~km} \mathrm{~s}^{-1}$, whereas Nidever et al. (2002) determine a radial velocity of $22.17 \mathrm{~km} \mathrm{~s}^{-1}$ for the primary $\mathrm{K}$ dwarf. The radial velocity for the WD companion is likely to include the gravitational redshift, which may account for the apparent difference with HIP 4849. We estimate an age of $1.3 \mathrm{Gyr}$ for HIP 4849 from a chromospheric activity index of $\log R_{\mathrm{HK}}^{\prime}=-4.661$ given by Gray et al. (2003), whereas the cooling age for WD $0101+048$ is $0.63 \pm 0.07 \mathrm{Gyr}$, not including its main-sequence lifetime (Bergeron et al. 2001).

The pair of stars HIP 50564 and NLTT 23781 is remarkable not least because of its extreme separation (5230", or 111,000 AU on the sky). Other interesting properties of this system are discussed in $\S 6.2$.

The CPM pair of HIP 22498 (DP Cam) and G247-35 is separated by "only" $1000 \mathrm{AU}$ in the sky projection, and it is surprising it has not been identified as such before. The primary component, a K7 dwarf, is listed as eclipsing binary in the catalog of eclipsing stars (Malkov et al. 2006). Its Hipparcos parallax is very poor even for a "stochastic" solution, indicating an unresolved type of binarity; however, both this binary and its distant M-type companion lie on the main sequence in Figures 1 and 2. Very little is known about $\mathrm{G}$ 247-35, apart from the photometric observations in (Weis 1988).

\section{ACTIVITY AND AGES \\ 6.1. Chromospheric Activity}

The so-called $\mathrm{H} \alpha$ limit relation tells us that there is a certain age in the evolution of $\mathrm{M}$ dwarfs of a given mass (or $V-I$ color) when the ubiquitous chromospheric activity, related by emission in the $\mathrm{H} \alpha$ line, disappears and the stars transform from dMe to normal inactive dwarfs (Gizis et al. 2002). The empirical relation, fairly well defined on open clusters, can be written as

$$
\log \operatorname{Age}_{\mathrm{H} \alpha}=0.952\left(V-I_{\mathrm{C}}+6.91\right) \text {. }
$$

This formula should be used with caution because recent studies of M dwarf activity based on large samples of stars selected from the Sloan Digital Sky Survey indicate that the activity lifetime versus spectral-type relation is strongly nonlinear (West et al. 2008), with a steep ascent between M3 and M5. This abrupt change may be related to the transition from partially convective to fully convective stellar interiors. Most of the latest $\mathrm{M}$ dwarfs in the solar neighborhood are active, but an age-activity correlation is still evident at spectral type M7 where the fraction of chromospherically active stars declines with the distance from the Galactic plane (West et al. 2006). This relation can be used to differentiate the oldest late-type $\mathrm{M}$ dwarfs, although exact calibration

TABLE 3

WDS Identifications and New Pairs

\begin{tabular}{|c|c|c|c|c|c|c|c|c|c|}
\hline HIP & WDS & HIP & WDS & HIP & WDS & HIP & WDS & HIP & WDS \\
\hline $473 \ldots \ldots \ldots . .$. & $00057+4549$ & 4849 & New & 4872 & New & 5799 & New & 9749 & $02053-2803$ \\
\hline $14286 \ldots \ldots$ & $03042+6142$ & 14555 & $03079-2813$ & 15371 & $03182-6230$ & 17414 & $03439+1640$ & 21482 & $04368+2708$ \\
\hline $22498 \ldots \ldots .$. & $04503+6320$ & 25278 & New & 34065 & $07040-4337$ & 42748 & $08427+0935$ & 43587 & $08526+2820$ \\
\hline $49669 \ldots \ldots$. & $10084+1158$ & 46843 & $09327+2659$ & 47620 & $09427+7004$ & 50564 & New & 59000 & New \\
\hline $59406 \ldots \ldots .$. & $12113-1958$ & 61451 & $12356-3453$ & 63882 & $13055+3708$ & 65083 & $13203-0140$ & 65877 & $13303-0834$ \\
\hline $66492 \ldots \ldots$. & $13379+4808$ & 71914 & $14426+1929$ & 75718 & $15282-0921$ & 79607 & $16147+3352$ & 82817 & $16555-0820\left(231^{\prime \prime}\right.$ comp. is new $)$ \\
\hline $83591 \ldots \ldots .$. & $17050-0504$ & 86036 & $17350+6153$ & 93899 & $19072+2053$ & 97295 & $19464+3344$ (792" comp. is new) & 98204 & $19573-1234$ \\
\hline $98767 \ldots \ldots$. & $20036+2954$ & 102409 & $20452-3120$ & 109084 & New & 113602 & New & 115147 & $23194+7900$ \\
\hline $116215 \ldots .$. & $23328-1651$ & & & & & & & & \\
\hline
\end{tabular}


TABLE 4

X-Ray Luminosities

\begin{tabular}{|c|c|c|}
\hline $\begin{array}{c}\text { HIP/Name } \\
\text { (1) }\end{array}$ & $\begin{array}{l}L_{\mathrm{X}} \\
(2)\end{array}$ & $\begin{array}{l}\text { HR1 } \\
\text { (3) }\end{array}$ \\
\hline 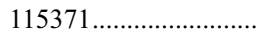 & $0.058 \pm 0.013$ & -0.91 \\
\hline 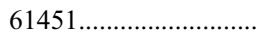 & $0.11 \pm 0.04$ & -0.86 \\
\hline 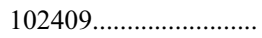 & $5.59 \pm 0.11$ & -0.07 \\
\hline 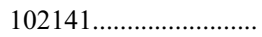 & $3.38 \pm 0.10$ & -0.19 \\
\hline 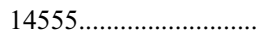 & $4.62 \pm 0.27$ & -0.27 \\
\hline 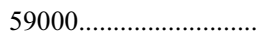 & $0.71 \pm 0.24$ & +0.20 \\
\hline 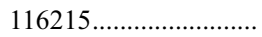 & $0.13 \pm 0.03$ & -0.56 \\
\hline 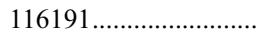 & $1.41 \pm 0.09$ & -0.28 \\
\hline $75722 \ldots \ldots$ & $0.43 \pm 0.07$ & -0.40 \\
\hline $82817 \ldots$ & $1.09 \pm 0.07$ & -0.26 \\
\hline $5799 \ldots \ldots$ & $0.74 \pm 0.12$ & -0.01 \\
\hline 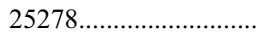 & $0.16 \pm 0.03$ & -0.48 \\
\hline 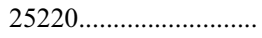 & $2.75 \pm 0.11$ & -0.12 \\
\hline 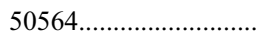 & $1.09 \pm 0.38$ & +0.22 \\
\hline 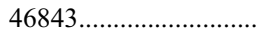 & $1.49 \pm 0.09$ & -0.19 \\
\hline $21482 \ldots \ldots \ldots \ldots \ldots \ldots \ldots \ldots \ldots \ldots \ldots \ldots \ldots$ & $8.20 \pm 2.38$ & -0.04 \\
\hline $97222 \ldots \ldots \ldots \ldots . . . . . .$. & $0.087 \pm 0.023$ & -0.85 \\
\hline LTT $15775 \ldots \ldots \ldots \ldots \ldots$ & $0.048 \pm 0.06$ & -0.29 \\
\hline 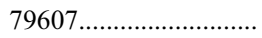 & $46.1 \pm 0.6$ & +0.06 \\
\hline 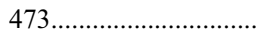 & $0.050 \pm 0.014$ & -0.42 \\
\hline 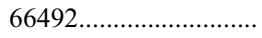 & $0.076 \pm 0.028$ & -0.74 \\
\hline 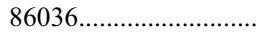 & $0.47 \pm 0.02$ & -0.48 \\
\hline $86087 \ldots \ldots \ldots \ldots \ldots \ldots$ & $0.028 \pm 0.005$ & -0.58 \\
\hline V388 Cas ....................... & $0.20 \pm 0.02$ & -0.19 \\
\hline 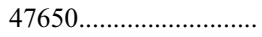 & $0.18 \pm 0.03$ & -0.40 \\
\hline 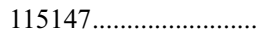 & $10.6 \pm 0.2$ & -0.10 \\
\hline
\end{tabular}

Notes._Col. (1): HIP number or name. Col. (2): X-ray luminosity in units of $10^{29} \mathrm{ergs} \mathrm{s}^{-1}$. Col. (3): Hardness ratio HR1 from ROSAT.

is currently problematic because of the lack of independent age estimates.

A widely used means of age estimation is provided by the empirical relation between the level of chromospheric activity as measured from the $R_{\mathrm{HK}}^{\prime}$ index of Ca II lines. The equation used in this paper,

$$
\log \mathrm{Age}_{\mathrm{HK}}=(-2.02 \pm 0.13) \log R_{\mathrm{HK}}^{\prime}-(0.31 \pm 0.63),
$$

was derived by (Soderblom et al. 1991) for the Sun, Hyades, and UMa Group. We utilize these relations in Table 5 to estimate (very roughly) the ages and age limits for several late-type components.

\subsection{X-Ray Activity}

The binary and multiple systems under investigation in this paper are so wide that the observed ROSAT sources can be unambiguously identified with individual components. Table 4 lists all the components identified by us in the ROSAT Bright Source and Faint Source catalogs (Voges et al. 1999, 2000). The hardness ratios HR1 in this table are from the ROSAT catalogs, while the $\mathrm{X}$-ray luminosities, in units of $10^{29} \mathrm{ergs} \mathrm{s}^{-1}$, are computed from the specified count rates, hardness ratios, and Hipparcos parallaxes. Most of the faint sources, with $L_{\mathrm{X}}<1$, are very soft, with HR1 closer to -1 . They are similar in X-ray activity to the quiescent Sun, or slightly exceed it. The vast majority of weak nearby dwarfs are likewise soft, indicating low coronal activity (Hünsch et al. 1999). Normal M-type dwarfs have significantly smaller X-ray luminosities than G- and K-type stars. Indeed, most of the X-ray-weak systems include a K-type primary, and a few F-type primaries, whereas numerous M-type wide companions are not detected by ROSAT. A few notable M-type emitters should be mentioned.

The star HIP 14555 is a flare M0 dwarf with a Hipparcos parallax of $\Pi=52 \pm 5$ mas. This poor parallax determination, in addition to a great deal of confusion associated with this multiple system, is related to a failed component solution in Hipparcos, based on the wrong assumption that HIP 14555 and HIP 14559 (at separation $30.3^{\prime \prime}$, position angle $101^{\circ}$ ) form a physical pair at the same distance from the Sun. Fabricius \& Makarov (2000a) resolved the Hipparcos data for this system using more accurate initial assumptions and obtained a parallax $\Pi=55.2 \pm 2.5$ mas and a proper motion $\mu=(-339,-121) \pm(2.5,2.2) \operatorname{mas} \mathrm{yr}^{-1}$ for HIP 14555, which is quite close to the original solution, but a

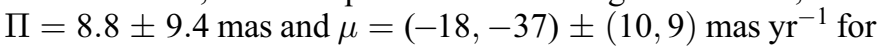
HIP 14559. Thus, these stars are certainly optical companions. LTT 1477 is probably a real, albeit more remote, CPM companion. The outstanding X-ray brightness of HIP 14555 finds explanation in the observations by Gizis et al. (2002), who find it to be a double-lined spectroscopic binary (SB2) with a remarkable surface velocity of rotation $v \sin i=30 \mathrm{~km} \mathrm{~s}^{-1}$. We are dealing with a typical extremely active $M$ dwarf in a multiple system: a short-period spectroscopic binary with a remote companion.

The star HIP 47650 is an M3 flare star and a member of the Hyades stream according to Montes et al. (2001). Nidever et al. (2002) determined a "stable" radial velocity of $+6.6 \mathrm{~km} \mathrm{~s}^{-1}$ for this star, precluding a detectable spectroscopic companion. We should therefore consider the possibility that this star is young. Both HIP 47650 and its brighter companion HIP 47620 lie significantly above the empirical main sequence in Figure 1. These stars are brighter in $M_{K s}$ than the empirical main sequence from Henry et al. (2004), by 0.71 and $0.76 \mathrm{mag}$, respectively. Figure 2 shows that both components are also brighter than their field counterparts in $M_{V}$ versus $V-K_{s}$ axes as well, but by a smaller amount ( 0.49 mag in both cases). These photometric data suggest a large $K$-band excess, probably due to a young age similar to the age of the Pleiades. The substantial amount of X-ray radiation from HIP 47650 is accompanied by pronounced chromospheric activity. According to Wright et al. (2004) its average $S$-value of $\mathrm{Ca}$ II chromospheric activity of 3.2 is outside and above the normal range where calibrated indices $R_{\mathrm{HK}}^{\prime}$ can be estimated. What remains puzzling is that two stars of similar mass in a binary system can be so different in chromospheric and coronal activity: HIP 47650 is a dMe star with $\mathrm{EW}_{\mathrm{H} \alpha}=2.87 \AA$ (Rauscher \& Marcy 2006), whereas HIP 47650 has no emission in $\mathrm{H} \alpha$ (Gizis et al. 2002). Since the difference in $V-I_{\mathrm{C}}$ between the components is only $0.2 \mathrm{mag}$, employing formally the age criterion in $\S 6.1$ places the system in very narrow brackets of age just above 1 Gyr. However, it seems unlikely that both chromospheric and X-ray activity in the more massive companion HIP 47620 waned so abruptly; the transition from $\mathrm{dMe}$ to normal $\mathrm{M}$ dwarfs is probably protracted and statistically uncertain. This binary system indicates that the evolution of surface rotation, which is a crucial factor in solarand subsolar-mass dwarfs, may take different courses even for coeval, nearly identical stars.

By far the brightest X-ray source in our collection is the BY Dra-type binary HIP 79607 ( TZ CrB, orbital period 1.14 days). This example confirms that short-period spectroscopic binaries with evolved or solar-type primaries are the most powerful emitters among normal stars, surpassing single pre-main-sequence stars in X-ray luminosity by a factor of a few (Makarov 2003). The impressive flare activity on this star was investigated in detail by Osten et al. (2000). Its distant companion HIP 79551 separated by at least 13,000 AU is an M2.5 dwarf without any signs of chromospheric activity; we surmise that it should be older than 
TABLE 5

Velocities, Moving Groups, Activity, and Ages

\begin{tabular}{|c|c|c|c|c|c|c|c|}
\hline HIP/Name & $U$ & $V$ & $W$ & SKG & References & $\mathrm{EW}(\mathrm{H} \alpha)$ & $\log \mathrm{Age}$ \\
\hline $15330 \ldots \ldots \ldots \ldots \ldots$ & -71 & -47 & +16 & Hercules? & 1 & & 9.2 \\
\hline $15371 \ldots \ldots \ldots \ldots \ldots$ & -70 & -46 & +16 & Hercules? & 1 & & 9.4 \\
\hline $47620 \ldots \ldots \ldots \ldots \ldots$ & -36 & -13 & -14 & Hyades & 2 & $<0$ & $>8.7$ \\
\hline $47650 \ldots \ldots \ldots \ldots \ldots$ & -35 & -13 & -14 & Hyades & 2 & 2.87 & $<8.9$ \\
\hline $79607 \ldots \ldots \ldots \ldots \ldots$ & -7 & -29 & +9 & & & 0.64 & $>9.5$ \\
\hline $21482 \ldots \ldots \ldots \ldots \ldots$ & -39 & -17 & -2 & Hyades? & 3 & 1.0 & $>9.6$ \\
\hline $102409 \ldots \ldots \ldots \ldots$ & -10 & -17 & -10 & BETAPIC & 4 & 2.2 & 7.0 \\
\hline $102141 \ldots \ldots \ldots \ldots$ & -9 & -16 & -11 & BETAPIC & 4 & 10.9 & 7.0 \\
\hline $25278 \ldots \ldots \ldots \ldots \ldots$ & -37 & -15 & 8 & Hyades & 2 & & 8.5 \\
\hline $25220 \ldots \ldots \ldots \ldots \ldots$ & -38 & -14 & 7 & Hyades & 2 & -0.76 & 8.7 \\
\hline $116215 \ldots \ldots \ldots \ldots$ & -13 & -21 & -10 & & & -0.59 & 8.8 \\
\hline $116191 \ldots \ldots \ldots \ldots$ & -13 & -21 & -10 & & & 1.98 & $<9.0$ \\
\hline $4872 \ldots \ldots \ldots \ldots \ldots \ldots$ & -32 & -16 & 6 & Hyades & 2 & & \\
\hline 43587................ & -37 & -18 & -8 & Hyades & 2 & & \\
\hline
\end{tabular}

REFERENCES.- (1) Soubiran \& Girard 2005; (2) Montes et al. 2001; (3) Eggen 1993; (4) Makarov 2007a.

3 Gyr $(\S 6.1)$, setting a lower bound on the age of the primary component. The primary, a F6 + G0 pair of dwarfs (Frasca et al. 1997), has a visual companion at 5.9", orbital period $852.8 \mathrm{yr}$ (Tokovinin et al. 2006). This inner companion $(\sigma 1 \mathrm{CrB})$ may be responsible for the tight spectroscopic pair via the Kozai cycle, if the original orbits were not coplanar $(\S 7.1)$. In this case, the substantial age of the system estimated from the CPM companion is consistent with the timescale of dynamical evolution. The vertical velocity component with respect to the local standard of rest is $+16 \mathrm{~km} \mathrm{~s}^{-1}$, assuming a standard solar velocity of $W=$ $+7 \mathrm{~km} \mathrm{~s}^{-1}$. This places the TZ CrB multiple system in the older thin disk (Table $2, \S 3$ ), whose constituents spend roughly onethird of their lifetimes in the dense part of the Galactic disk. Thus, survival of the wide companion for longer than $3 \mathrm{Gyr}$ is plausible.

The star HIP 21482 appears to be another example of an extremely active BY Dra-type spectroscopic binary in a hierarchical multiple system (Tokovinin et al. 2006). The inner spectroscopic pair has a orbital period of 1.788 days and is already circularized and rotationally synchronized (Montes et al. 1997). Its heliocentric motion (Table 5) is similar to the Hyades stellar kinematic group (SKG), except for the deviating, small $W$ velocity. The star was even suspected of having originated in the Hyades open cluster, which would fix its age at $600 \mathrm{Myr}$; in particular, Eggen (1993) suggested that it could belong to the extended halo of evaporated stars around this cluster. The exceptional chromospheric activity of the inner pair at $\log R_{\mathrm{HK}}^{\prime}=-4.057$ at the very tail of the distribution observed for nearby field stars (Gray et al. 2003), may also indicate a young age. However, the remote companion WD $0433+270$ is a cool DC white dwarf, and therefore the system can hardly be young. Bergeron et al. (2001) estimated a $T_{\text {eff }}=5620 \pm 110 \mathrm{~K}$ and cooling age of $4.07 \pm 0.69 \mathrm{Gyr}$, an order of magnitude older than the Hyades.

The star HIP 115147 (V368 Cep) is one of the nearest post$\mathrm{T}$ Tauri stars. It is mistakenly identified as a RS CVn-type binary in the SIMBAD database, although, contrary to the previously discussed objects of this type, it is not a spectroscopic binary. Both its secondary companion at $11^{\prime \prime}$ and the newly discovered tertiary CPM companion LSPM J2322+7847 (Makarov et al. 2007) lie significantly above the main sequence in optical and infrared colors. The probable age of this system is only $20-50 \mathrm{Myr}$, and the high rate of rotation of the primary (with a period of 2.74 days; Kahanpää et al. 1999) is obviously due to its youth. The origin of this post-TT triple system is an open issue, a high-velocity ejection from the Ophiuchus SFR being one of the possibilities considered.

The pair of outstanding T Tauri stars HIP 102409 (AU Mic) and HIP 102141 (AT Mic) epitomize the class of very young, active X-ray emitters. They may be as young as $10 \mathrm{Myr}$, and both display the whole complement of stellar activity indicators. AU Mic has a nearly edge-on debris disk, and its remarkable X-ray luminosity is probably nurtured by the high rate of rotation with a period of surface spots of 4.847 days (Hebb et al. 2007). Its distant companion, AT Mic, is a flare M4.5 dwarf and an extreme UV source. Both stars lie significantly above the $16 \mathrm{Myr}$ isochrone in Figure 1. AT Mic has a somewhat poorly investigated companion LTT 8182 at $3.8^{\prime \prime}$, position angle $218^{\circ}$ which is missing in the 2MASS survey and omitted in Table 1. Its $\mathrm{H} \alpha$ emission is also remarkably high ( $\mathrm{EW}=9.3 \AA$; Scholz et al. 2007). AT Mic and AU Mic are separated by more than $0.2 \mathrm{pc}$ in the sky plane, one of the largest separations found in this paper, and it is unlikely the two stars could be gravitationally bound. They will inevitably part ways in the future, along with other members of the dispersed BETAPIC stream (Makarov 2007a).

Both components in the CPM pair HIP 25278 and 25220 are prominent X-ray sources (Hünsch et al. 1999). The primary GJ 202, an F8 V star, is, however, more than 10 times weaker than its K4 companion GJ 201. Both stars have been assigned to the Hyades SKG by Montes et al. (2001). HIP 25278 appears to be a single star of slightly subsolar metallicity with an estimated age of 5.6 Gyr (Nordström et al. 2004). Takeda \& Kawanomoto (2005) determine a slightly higher $[\mathrm{Fe} / \mathrm{H}]=0.05$ and find a surprisingly high content of lithium $(\mathrm{EW}=0.094 \AA$ ). Another unexplained characteristic of this star is its position below the main sequence in Figure 1 . The moderate $\mathrm{X}$-ray activity is accompanied by a noticeable Ca II chromospheric signature at $\log R_{\mathrm{HK}}^{\prime}=-4.38$ and rotation $P / \sin i=4.1$ days (Reiners \& Schmitt 2003). Using the above value for $\log R_{\mathrm{HK}}^{\prime}$ and equation (7), we obtain an age of 0.3 Gyr (Table 4), significantly less than Nordström et al.'s estimate, and roughly consistent with the age of the Hyades open cluster. The CPM companion GJ 201, an active K4 V star, has a $\log R_{\mathrm{HK}}^{\prime}=-4.452$ (Gray et al. 2003), and hence, an age of 0.48 Gyr. It appears to be spectroscopically single. Its lithium abundance is low, however (Favata et al. 1997). Furthermore, the $\mathrm{H} \alpha$ line is in absorption according to Herbst \& Miller (1989) placing this star in the realm of inactive, regular dwarfs. The high level of X-ray activity in this stars remains a mystery, because it cannot be explained just by the relative youth. Indeed, the distribution of X-ray luminosity between the companions appears to be inverted to that observed in the Hyades cluster (Stern et al. 1995), in that the F8 primary companion is below the lower envelope of $L_{\mathrm{X}}$ for its Hyades counterparts, while the secondary component, GJ 201, is roughly a factor of 10 more luminous than the average $\mathrm{K}$ dwarf in the Hyades and is comparable in X-ray emission to the brightest nonbinary F8-G0 Hyades members.

The stars HIP 116215 and 116191, of spectral types K5 and M3.5, respectively, have space velocities similar to the Local stream of young stars (Montes et al. 2001). They may be as young as the Pleiades. The primary component (GJ 898) is single and its $\mathrm{X}$-ray luminosity is similar to the average value for the Hyades late K-dwarfs. The secondary (GJ $897 \mathrm{AB}$ ) is a resolved visual binary (Mason et al. 2002) with an orbital period of $28.2 \mathrm{yr}$ and a semimajor axis of $0.59^{\prime \prime}$, which probably explains why this $\mathrm{M}$ dwarf lies significantly above the main sequence, while the primary is quite close to it. A $\log R_{\mathrm{HK}}^{\prime}=-4.486$ from Gray et al. (2003) for HIP 116215 implies an age of $560 \mathrm{Myr}$, again similar to the age of the Hyades. The $\mathrm{H} \alpha$ line is in absorption for the 
primary, but prominently in emission for the secondary (Gizis et al. 2002). This fact can be used to estimate the boundaries of $\mathrm{H} \alpha$-age (§ 6.1), which yields log (Age) $\in[8.1,9.0]$, in good agreement with the $\log R_{\mathrm{HK}}^{\prime}$-age estimate. The remaining difficulty in the interpretation of this system is the unusual strength of X-ray emission from HIP 116191, by far surpassing the levels observed for this age and spectral type in the Hyades. One may suspect that one of the visual companions in this binary is an undetected shortperiod spectroscopic binary.

The star HIP 46843 is likely another representative of young X-ray emitters. A $\log R_{\mathrm{HK}}^{\prime}=-4.234$ from Gray et al. (2006) yield an age of $175 \mathrm{Myr}$, in fine agreement with the rotational age estimate 164 Myr from Barnes (2007). Its M5.5 companion GJ 9301 B is undetected in X-rays. The young age of this system is confirmed by the $L_{X}$ in Table 4 for HIP 46843, which is only slightly smaller than the typical luminosity of Pleiades members $\left(\sim 3 \times 10^{29}\right.$; Stauffer \& Hartmann 1986) of this spectral type. GJ 9301 B is therefore one of the youngest late $\mathrm{M}$ dwarfs in the solar neighborhood. Note that SIMBAD mistakenly provides an uncertain estimate of $M_{V}$ from (Reid et al. 1995) as a $V$ magnitude.

The star HIP 50564 of spectral class F6IV is remarkably active in X-ray but is unremarkable chromospherically $\left(\log R_{\mathrm{HK}}^{\prime}=\right.$ -4.749; Gray et al. 2003) and depleted in lithium. The low degree of activity points at an age of $1.9 \mathrm{Gyr}$. On the other hand, this star is a $\delta$ Scuti-type variable and a fast rotator, $v \sin i=17 \mathrm{~km} \mathrm{~s}^{-1}$. It has a solar iron abundance, $[\mathrm{Fe} / \mathrm{H}]=0.09$ from (Nordström et al. 2004) and a space motion typical of the local young stream, $(U, V, W)=(-14,-26,-12) \mathrm{km} \mathrm{s}^{-1}$. The key to the mystery of its X-ray activity may be in a short-period, low-mass companion; indeed, Cutispoto et al. (2002) mention that the star is "reported as SB1" (single-lined spectroscopic binary) without providing further detail. Its M5-type CPM companion NLTT 23781 separated by at least $0.5 \mathrm{pc}$ is one of the discoveries in this paper. It was cataloged in (Lépine \& Shara 2005; Salim \& Gould 2003), but otherwise, this interesting object completely escaped the attention of observers. Its location in the HR diagram (Fig. 1) above the main sequence indicates a young age or binarity. Thus, this extreme system represents a mystery in itself. If it is indeed 1.9 Gyr old, how could it survive at this separation having spent all the time in the thin disk, and why the remote companion is overluminous?

The star HIP 59000 has a known CPM companion NLTT 29580 separated by $4200 \mathrm{AU}$ in the sky projection. Gray et al. (2006) report a substantial chromospheric activity of the primary, $\log R_{\mathrm{HK}}^{\prime}=-4.341$, which translates into a chromospheric age of 0.29 Gyr. HIP 59000 is orbited by a low-mass companion, probably a brown dwarf, for which we derive a first orbital solution in $\S 7$. This inner companion is not close enough to the primary ( $P \simeq 5.1 \mathrm{yr}$ ) to account for the significant $\mathrm{X}$-ray luminosity of the system. We think that either the primary is a yet-undetected short-period spectroscopic binary (in which case the astrometric companion may have a stellar mass), or the system is indeed fairly young. The remote CPM companion NLTT 29580, a M5.0 star, is confirmed the photometric parallax from Reid et al. (2003) being in excellent agreement with the updated parallax of HIP 59000 (45 mas).

There is little doubt that the origin of the X-ray activity in HIP 82817 is in the innermost component of this intriguing system of at least five stars, which drives the fast rotation of the secondary. Indeed, the A component is orbited by a B component at $P_{\mathrm{AB}}=626$ days, which is in fact some $50 \%$ more massive than the primary because it is a spectroscopic binary with a period $P_{\mathrm{B}}=2.96553$ days and a mass ratio of 0.9 (Mazeh et al. 2001), made of nearly identical $\mathrm{M}$ dwarfs. Both eccentricities are low, and the orbits are likely to be coplanar. The widest CPM companion LHS 429 is a M7 dwarf lying on the empirical main sequence for late field dwarfs (Fig. 1). Mazeh et al. (2001) suggest an age of $\sim 5$ Gyr for the system.

The F5 V star HIP 5799 and its G9 CPM companion GJ 9045 B are moderately metal-deficient $([\mathrm{Fe} / \mathrm{H}]=-0.3)$, kinematically belong to the thin disk population and have an estimated age of 2.5 Gyr (Soubiran \& Girard 2005). This age estimation is supported by the moderate HK activity obtained by Gray et al. (2003) for the primary. The combination of a significant X-ray emission from the primary and the lack of such from the secondary, a modest rotational velocity of HIP $5799\left(v \sin i=4.4 \mathrm{~km} \mathrm{~s}^{-1}\right.$; Tokovinin 1990) and the above age are puzzling. The peculiar location of HIP 5799 in the HR diagram (Fig. 1) much above the main sequence and closer to the $16 \mathrm{Myr}$ isochrone may give a clue. This star may be a yet undetected short-period spectroscopic binary seen almost face-on.

The CPM pair HIP 86036 (=26 Dra) and HIP 86087 (=GJ 685) represents a rare case when both components are detected by ROSAT. Their X-ray luminosities differ by more than a factor of 10 which may be the natural consequence of the difference in the sizes of their coronae, the primary being a G0 V star and the distant companion a M1 V dwarf. The primary is in fact a triple system where A component has a 76 yr orbiting $\mathrm{B}$ companion and a wide low-mass C companion at 12.2" (Tokovinin et al. 2006). Definitely, these resolved companions (not present in our sample) are not responsible for the enhanced X-ray emission from the inner system and we have to look for signs of a young age. Surprisingly, we find conflicting data. The primary star HIP 86036 is moderately metal-poor $([\mathrm{Fe} / \mathrm{H}]=-0.18)$ and has an age of $8.4 \mathrm{Gyr}$ according to Soubiran \& Girard (2005). Nordström et al. (2004) give an even older age of $11.5 \mathrm{Gyr}$ for this star. However, the rotational age of the distant companion GJ 685 is only $435 \pm 50 \mathrm{Myr}$ at $P_{\text {rot }}=18.6$ days (Barnes 2007). The $\mathrm{H} \alpha$ line is in absorption (EW $=-0.4 \AA$; Stauffer \& Hartmann 1986), which only means that this M1V star is probably older than 200 Myr. Another confusing detail comes from the $\mathrm{Ca}$ II $\mathrm{HK}$ line flux which is low for this type of star and the period of rotation (Rutten 1986). It is possible that the fast rotation of GJ 685 is driven by extraneous agents, and the rotation age estimate is confused. To summarize, the origin of X-ray activity and the age of this system remains unknown.

\section{MULTIPLICITY}

At least 17 out of our 41 CPM systems contain inner binary or triple components. We have reasons to believe that some of the CPM components are still undiscovered binaries, especially those stars that are too luminous for their spectral type and age, and have enhanced rates of rotation and chromospheric activity. The rate of triple and higher order multiple systems among nonsingle stars in the Hyades is only 0.14 (Patience et al. 1998), significantly smaller than we find for CPM pairs (0.41). To some extent, the high-order multiplicity of very wide pairs can be explained by the higher mass of binary stars and therefore, better chances of survival in the course of dynamical interaction with other constituents of the Galaxy. This argument may be particularly relevant for older CPM systems of extreme separations. On the other hand, there may be a more subtle reason for the abundance of hierarchical systems. The primary fragmentation of a prestellar molecular cloud and the secondary fragmentation during $\mathrm{H}_{2}$ dissociation are likely to take place at two distinct hierarchical spatial scales (Whitworth \& Stamatellos 2006). Of the three main models of low-mass star formation considered in that paper, the two-dimensional fragmentation triggered by supersonically colliding gas streams appears to be the most plausible scenario for wide companions in multiple 
systems. It predicts a wide range of initial orbital eccentricities and relative inclinations in such systems.

Perhaps the system of CPM companions HIP 473 and 428 is the most important for empirical study of the Kozai-type evolution of multiple systems. The latter star, an M2e dwarf, is known as the F components of the system $\operatorname{ADS} 48$, where the primary star has a visual twin companion B (spectral type M0) separated by $6^{\prime \prime}$. The most interesting aspect of this system is that the mutual inclination of the $\mathrm{B}$ and $\mathrm{F}$ companions is $\simeq 80^{\circ}$ according to the family of probable orbits computed by Kiyaeva et al. (2001). The eccentricity of the inner pair $\mathrm{AB}$ is probably between 0.2 and 0.6. Therefore, ADS 48 may be a paragon of the Kozai evolution in progress, where the inner pair has not yet shrunk but remains in an elliptical orbit. Kiyaeva et al. (2001) note a probable inner tertiary companion, which may account for the total dynamical mass higher by $\sim 0.3 M_{\odot}$ than what is expected from the spectral type. Furthermore, they note a slight variation in position of the A component with a period of $15 \mathrm{yr}$, possibly indicating another $\simeq 0.05 M_{\odot}$ companion. The A component lies on, or slightly below, the main sequence in Figure 2; thus, the hypothetical companions contribute little in the total luminosity. Anosova \& Orlov (1991) pointed out that the probability of a hyperbolic orbit for the F component appears to be greater than of an elliptical orbit. Such systems may be unstable in the long run. In the latter paper, it is proposed that ADS 48 is a member of the Hyades flow, of which we have quite a few representatives in our selection (Table 5). Stars in a kinematically coherent stream are more likely to be found in accidental slow passages near each other. The star HIP 428 is an emission-line M2 dwarf (Rauscher $\&$ Marcy 2006), indicating an upper limit on age of $\simeq 1$ Gyr. This estimate is consistent with the upper envelope of the Hyades flow (Eggen 1998). We believe that Kiyaeva et al.'s suggestion that the F companion is physically bound to the $\mathrm{AB}$ pair with an orbital period of $\sim 10^{5} \mathrm{yr}$ is more plausible in the light of recent astrometric data.

The nearby star HIP 14555 (GJ 1054A), along with its optical companion HIP 14559, epitomizes the difficulties that arise in the reduction of Hipparcos data for visual multiple systems (§ 6.2). The improved solution for HIP 14555 from Fabricius \& Makarov (2000a) is $\Pi=55.5 \pm 2.5 \mathrm{mas},\left(\mu_{\alpha} \cos \delta, \mu_{\delta}\right)=(-339$, $-121)$ mas $^{-1}$, which is close to the original results. The remaining inconsistency is that with the estimation by Henry et al. (2002) who inferred a distance of $12.9 \mathrm{pc}$ based on their spectral type determination and the $V$ magnitude specified in Hipparcos. This biased estimate comes from the photometric data which seem to be too bright. Figure 2 depicts the HR diagram for both HIP 14555 and the alleged CPM companion LTT 1477, with photometric data from (Weis 1993) and the same parallax of 55.5 mas assumed for both stars. The primary component lies significantly above the empirical main sequence and appears to match the $16 \mathrm{Myr}$ isochrone from (Siess et al. 2000). Despite the prominent $\mathrm{H} \alpha$ emission and X-ray activity, this star is not considered to be young. The apparent brightness excess is the consequence of unresolved binarity of HIP 14555. Indeed, according to Gizis et al. (2002), the star is double-lined spectroscopic binary (SB2).

The star HIP 34052 (GJ 264) is the tertiary component of a well-known wide triple system, which also include the pair of solar-type stars GJ 9223 (A) and GJ 9223 (B), separated by $21^{\prime \prime}$ on the sky. By virtue of the high proper motion and brightness, the system has been included in the lists of nearby star for a long time, attracting considerable attention due to the possibility of testing the evolution of stellar gravity, temperature and chemical composition in great detail. The spectroscopic investigation of components A and B by Chmielewski et al. (1991) found a common iron abundance of $[\mathrm{Fe} / \mathrm{H}]=-0.27 \pm 0.06$ and effective temperatures $5870 \pm 40$ and $5290 \pm 70 \mathrm{~K}$, respectively. Somewhat different lithium abundances were determined for the two components, but both at the solar level or below it. These estimates, together with the Galactic orbit (eccentricity 0.31 ) and a negligible chromospheric activity from the $\mathrm{Ca}$ II lines, indicate an old system, probably representing the old disk. A theoretical ZAMS used by Chmielewski et al. (1991) adjusted to the location of the B component on a $\log T_{\text {eff }}-M_{\text {bol }}$ diagram yielded a parallax of 68 mas. The trigonometric parallax of the system is close to 60 mas (Table 1). It may be suspected that the B component is too bright for the estimated $T_{\text {eff }}$ and metallicity. However, all three companions lie close to the main sequence with their photometric parameters in Table 1.

Relatively little is known about the tertiary component, HIP 34052. A robust astrometric solution was produced for this star in Hipparcos, without any indications of binarity or variability. However, the Hipparcos proper motion $\left(\mu_{\alpha} \cos \delta, \mu_{\delta}\right)=$ $(-75.4,401.3)$ mas $\mathrm{yr}^{-1}$ differs significantly from the Tycho-2

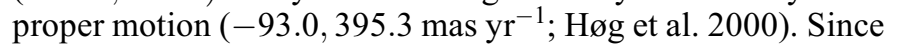
the latter is systematically more accurate in the presence of orbital motion, Makarov \& Kaplan (2005) included it in the list of astrometric binaries with variable proper motion. We further elaborate on this star by applying a multiparameter orbital optimization algorithm designed for the Hipparcos Intermediate Astrometry Data (HIAD) (see, e.g., Makarov 2004). This algorithm, based on the Powell method of nonlinear iterative optimization, looks for the global minimum of the $\chi^{2}$ statistics on abscissae residuals specified in the HIAD, corresponding to a certain combination of 12 fitting parameters, including seven orbital elements and five astrometric corrections. The estimated orbital parameters are period $P=1501$ days, inclination $i=180^{\circ}$, and apparent semimajor axis $a_{0}=30.6$ mas. The formal $F$-test on reduced $\chi^{2}(0.933$ after orbital adjustment) equals 1.0. The orbit is incomplete, because the period is longer than the time span of Hipparcos observations. Therefore, the orbital elements are fairly uncertain, and follow-up observations are needed to estimate the mass of the system. Assuming that the total mass of the system is $1.0 M_{\odot}$, the companion mass is only $0.2 M_{\odot}$, and the angular separation is about 150 mas $(a=2.6 \mathrm{AU})$. The companion may be possible to resolve with the Hubble Space Telescope (HST) or ground-based coronographic facilities.

Astrometric binarity of the HIP 59000 (GJ 9387) K7 dwarf is advertised by its varying proper motion (Makarov \& Kaplan 2005). It is not a known spectroscopic binary; therefore, we attempted an unconstrained 12-parameter orbital solution for this star using the same algorithm described in the previous paragraph. A visual inspection of the HIAD data reveals that the orbital period is several years, and we are dealing with another incomplete orbit. As a consequence, the fitted parameters should be considered preliminary. We obtain a period $P=1854$ days, apparent semimajor axis $a_{0}=12$ mas, $T_{0}=\mathrm{JD} 2,448,368, \omega=61^{\circ}, \Omega=53^{\circ}$, inclination $i=74^{\circ}$, and eccentricity $e=0.6$. The updated parallax is $\Pi=45.5 \pm 0.7$, which is close to the original Hipparcos parallax. The standard error of $a_{0}$ is about 2 mas, but the eccentricity is quite uncertain. Assuming a mass of $0.5 M_{\odot}$ for the visible primary, its apparent orbit on the sky leads to a total $a=2.34$ AU and a secondary mass of $0.063 M_{\odot}$. The expected radial velocity semiamplitude is $1.9 \mathrm{~km} \mathrm{~s}^{-1}$. Thus, this newly discovered binary system contains a brown dwarf which may be only 290 Myr old ( $(6.1)$.

The star HIP 75718 (GJ 586 A) is the primary in a system of at least four components (Tokovinin et al. 2006). The system is enshrouded in puzzles. The inner pair is both spectroscopic and astrometric (Duquennoy et al. 1992; Jancart et al. 2005) yielding a fairly detailed orbit. It consists of a K2 V dwarf (mass $0.74 M_{\odot}$ ) 
and a later $\mathrm{K}$ dwarf (mass $0.49 M_{\odot}$ ) in a 889.6 day orbit. The orbit has an outstanding eccentricity of $0.9752 \pm 0.0003$, so that the separation between the companions at periastron is only about 10 solar radii. The tertiary companion HIP 75722 (=GJ $586 \mathrm{~B})$ is separated by $52^{\prime \prime}$ in the sky projection. It is another K2 V dwarf of the same mass as the primary of the inner pair $\left(0.74 M_{\odot}\right)$. Hünsch et al. (1999) assign the considerable X-ray flux detected by ROSAT to both $\mathrm{A}$ and $\mathrm{B}$ components, but in our opinion, it is the B component, surprisingly enough, that is responsible for the X-ray emission (Table 4). The two companions are disparate in their $\mathrm{Ca}$ II line activity too, the A component being at $\log R_{\mathrm{HK}}^{\prime}=-4.97$, indicating an old star, and $\mathrm{B}$ at $\log R_{\mathrm{HK}}^{\prime}=-4.37$ (Wright et al. 2004). Formally, we would estimate the chromospheric age ( $(7.1)$ at 0.33 Gyr. Furthermore, the A and B components have different rates of rotation, $P_{\text {rot }}=39.0$ and 9.0 days, respectively. What could be the reason for the high activity and fast rotation of GJ 586 B? Tokovinin (1991) reported outlying radial velocity measures (spikes) for this star in otherwise constant series of observations and suggested that the $\mathrm{B}$ component can also be a high-eccentricity spectroscopic binary. If this is the case and the orbital period is of order a few days, the discrepant activity levels and age estimates are explained. However, Nidever et al. (2002) report a constant radial velocity from their extensive measurements. To confuse the matter more, Nordström et al. (2004) specify a fairly low probability (0.285) of constant radial velocity from their 18 observations spanning 6014 days. Finally, it is not clear whether the mysterious fourth component GJ $586 \mathrm{C}(\mathrm{G}$ 151-61) is physically associated with this triple or quadruple system. Its trigonometric parallax (Dahn et al. 1982) is tantalizingly close ( $\Pi=47 \pm 5$ mas), but the proper motion is $\sim 15 \%$ smaller. NOMAD supplies us with the following data for this star: position R.A. $=15^{\mathrm{h}} 27^{\mathrm{m}} 45.08^{\mathrm{s}}$, decl. $=$ $-9^{\circ} 01^{\prime} 32.5^{\prime \prime}(\mathrm{J} 2000.0)$, and proper motion $\mu=(30,-312) \pm$ $(2,3) \mathrm{mas} \mathrm{yr}^{-1}$. The available magnitudes are $V=15.41, J=$ $10.55, H=9.92$, and $K_{s}=9.63$. The smaller proper motion of this late M dwarf accounts for its absence in our NOMAD-based sample. Since the system appears to be genuinely old, it is doubtful that GJ $586 \mathrm{C}$ can form a kinematic group with the brighter counterparts.

\subsection{Candidate Stars with Planets}

It is commonly accepted that planets can be present in binary stellar systems. The latest investigations in this area indicate that $23 \%$ of candidate exosolar planetary systems also have stellar companions (Raghavan et al. 2006). Planets can form in stable circumbinary disks if the latter are large enough, so that the stellar binary and the distant planet form a dynamically stable hierarchical system. In very wide CPM systems, we encounter a different hierarchical composition, when the remote tertiary companion has a stellar mass. Such planetary systems may be subject to the longterm oscillatory perturbations of inclination and eccentricity over a long time (several gigayears) because of the secular loss of orbital energy known as the Kozai cycle. The eccentricity variation is especially important for the dynamical evolution of the inner planetary system. The Kozai-type variation is significant only if the tertiary companion has a different initial inclination from the inner orbit (Malmberg et al. 2007). If, for example, the initial inclination of the tertiary is $76^{\circ}$, the planet will periodically describe an orbit of $e=0.95$. This high eccentricity entails very close periastron passages of the primary. Giant gaseous planets will be subject to the tidal friction at periastron passages quite similar to the mechanism suggested for stellar binaries (Kiseleva et al. 1998). The gradual loss of angular momentum may lock the planet on a high-eccentricity orbit, resulting in secular shrinkage of the orbit. The orbits of very short-period "hot Jupiters"
( $P<10$ days) should be circularized similarly to tight spectroscopic binaries. It is also important to note that the dynamical evolution due to the Kozai mechanism may be quite different for single planets and stable planetary systems even if the initial inclination of the tertiary stellar companion is high. Innanen et al. (1997) point out that a system of four major solar system planets would remain stable and roughly coplanar in the presence of a distant companion on timescales much longer than the timescale of the Kozai cycle, owing to the mutual dynamical interaction between the planets.

Our sample of CPM systems includes two candidate exoplanet hosts. The star HIP 43587 (GJ 324, 55 Cnc), which has a comoving companion LTT 12311 at a projected separation of $1050 \mathrm{AU}$, is a solar-type dwarf suspected of bearing a system of at least four planets (McArthur et al. 2004). One of them (55 Cnc d) is a superJupiter with a mass $M \sin i=3.9 M_{\mathrm{J}}$, a period of about 5550 days and a semimajor axis of nearly 6 AU. The other three suggested planets have masses between 0.037 and $0.83 M_{\mathrm{J}}$ and periods ranging 2.8 to 44 days. The spectroscopically determined eccentricities are all small $(<0.1)$. There are a few conflicting clues about the age of the stellar components. The star $55 \mathrm{Cnc}$ lies above the empirical main sequence by $0.55 \mathrm{mag}$ according to (Butler et al. 2006). Both this star and its companion GJ 324 B lie slightly above the main sequence in the $M_{K s}$ versus $V-K_{s}$ diagram in Figure 1 . The primary has a moderately enhanced metallicity $[\mathrm{Fe} / \mathrm{H}]=0.315$, common among exosolar planet hosts. However, the chromospheric activity of $55 \mathrm{Cnc}$ is quite low, at $\log R_{\mathrm{HK}}^{\prime}=$ -5.04 (Wright et al. 2004), which is in fact close to the mean chromospheric flux parameters for the most inactive field solartype dwarfs (Gray et al. 2003). Wright et al. estimate a log (age) = 9.81, and indeed, a similar age of 9.87 ( $7 \mathrm{Gyr}$ ) is obtained from the HK index. Montes et al. (2001) list $55 \mathrm{Cnc}$ as a member of the populous Hyades stream (or kinematic group), based on its heliocentric velocity vector (see Table 5). In the light of recent investigations, the Hyades stream, originally believed to originate from the evaporating Hyades supercluster (Eggen 1993) of approximately $700 \mathrm{Myr}$ of age, incorporates stars of a wide range of age and chemical composition, indicating a curious phenomenon of dynamical alignment (Famaey et al. 2005).

The star HIP 98767 (GJ $777 \mathrm{~A}$ ) is similar to $55 \mathrm{Cnc}$ in metallicity $([\mathrm{Fe} / \mathrm{H}]=0.213)$ and brightness excess $\left(\Delta M_{V}=0.66\right)$ according to Butler et al. (2006). Both this star and its distant CPM companion LTT 15865, separated by more than 2800 AU, lie slightly above the main sequence in Figure 1, but perfectly on the empirical main sequence in Figure 2. The primary star lies above the main sequence by 0.66 in absolute $V$ magnitude according to (Butler et al. 2006) and is moderately metal-rich, $[\mathrm{Fe} / \mathrm{H}]=0.213$. We do not know how to interpret these discordant photometric data, except to assume that there is some anomaly in the $B$ and $K$ bands. The primary is suspected to bear not just a single planet but a system of at least two planets (Vogt et al. 2005), a short-period HD $190360 \mathrm{c}$ of mass $M \sin i \approx 0.06 M_{\mathrm{J}}$ and $P=17$ days, and again a Jupiter-like HD 190360 b of mass $M \sin i \approx 1.55 M_{\mathrm{J}}$ and orbital period 2900 days. The eccentricities are $\approx 0$ and 0.36 , respectively.

\section{MOVING GROUPS AND STREAMS}

The solar neighborhood is permeated with SKGs, which are evident as number density clumps in the three-dimensional velocity space (Chereul et al. 1998). Since these streams are only loosely coherent kinematically and are not supposed to be gravitationally bound, their existence poses a certain problem of dynamics. The Hyades stream figures prominently in our sample (Table 5). The Sun is located inside the Hyades stream today (but 
does not belong to it), so that any selection of the nearest stars will give preference to this SKG, as opposed to, for example, the Ursa Major SKG. Still, the large number of CPM systems in the Hyades stream is surprising for the following reason. Recent investigations indicate that the Hyades SKG is composed of stars and clusters of disparate ages and origins (Famaey et al. 2005), contrary to the previous hypothesis that it is the result of dynamical evaporation of a massive open cluster. But if this stream is purely dynamical phenomenon, a kind of focusing taking hold of random unrelated objects, why do we find so many CPM pairs which are apparently generic? A dynamical agent sufficiently powerful to rearrange the local six-dimensional phase space of the Galaxy would probably accelerate the disruption of wide binaries rather than preserve them. Furthermore, the possible Hyades stream members present in our sample do not look like random field stars. Many of them have enhanced levels of chromospheric and X-ray activity indicative of moderately young age ( $\sim 1$ Gyr, roughly consistent with the age of the Hyades open cluster). On the other hand, the presence of weakly bound CPM pairs in the Hyades SKG is not consistent with the dynamical evaporation paradigm, because the latter assumes a dynamical relaxation and ejection event. A pair of M dwarfs like HIP 47620 and 47650 (discussed in $\S 6.2$ ) is unlikely to be thrown out of the Hyades cluster and remain intact.

The star HIP 4872 and its distant companion V388 Cas (GJ 51) are related to the Hyades stream by Montes et al. (2001). The latter companion is a well-known M5 flare dwarf of considerable X-ray luminosity (Table 4) and EUV activity (Christian et al. 2001). A better age estimate can be obtained for the former companion, which is a flare M1.5 dwarf. Rauscher \& Marcy (2006) list this star as dMe with an $\mathrm{H} \alpha$ equivalent width of $2.0 \AA$. This yields an upper age limit of $280 \mathrm{Myr}$. The young age and the activity levels are consistent with this system being in the young core of the Hyades flow.

The pair of CPM companions HIP 15330 and $15371\left(\zeta^{1}\right.$ and $\zeta^{2}$ Ret) is remarkable because both stars lie significantly below the ZAMS for $Z=0.01$. They could be suspected to be metalpoor, but the iron abundance is only moderately low at $[\mathrm{Fe} / \mathrm{H}]=$ $-0.22 \pm 0.05$ according to del Peloso et al. (2000). The pair was originally assigned to the $\zeta$ Her SKG, but since the latter star does not appear to belong to the moving group, it was renamed to $\zeta$ Ret SKG. Lately, Soubiran \& Girard (2005) determined somewhat smaller iron abundances $(-0.34$ and -0.30$)$ for the two stars and assigned them to the Hercules SKG. The vertical velocity is $W=16 \mathrm{~km} \mathrm{~s}^{-1}$, the maximum excursion from the plane $z_{\max }=0.31 \mathrm{kpc}$, and the eccentricity of the Galactic orbit $e=$ 0.26 . Allen \& Herrera (1998) propose to define the thick disk as either $e \geq 0.3$ or $\left|z_{\max }\right|>400 \mathrm{pc}$. Thus, the CPM pair in question does not qualify for the thick disk by any of these kinematic criteria. The origin of this system and its peculiar blueness remains an unresolved issue.

\section{DISCUSSION}

One of the most interesting results of this paper is that we find little, if any, presence of a thick-disk or halo population in the local sample of very wide binaries. The only CPM system that may belong to the thick disk is the WD+dM4.5 pair HIP 65877 (DA3.5 white dwarf WD 1327-083) and LHS 353 (see Silvestri et al. 2002). This shows that even the widest pairs at separations greater than 1000 AU can survive for $\simeq 1$ Gyr staying constantly in the thin disk of the Galaxy, despite numerous encounter and dynamical interaction events. This observation does not refute the dynamical analysis presented in $\S 3$, because thick disk and halo stars are very rare in the solar vicinity, and our sample (based on bright stars in the Hipparcos catalog) is probably too small and incomplete to accommodate sufficient statistics. But if we boldly extrapolate this result to a wider part of the Galaxy, we conclude that normal thin-disk, moderately young or very young, stars dominate wide CPM binary and multiple systems. Statistically, this is quite consistent with the estimation by Bartkevičius $\&$ Gudas (2002) on a larger sample of 804 Hipparcos visual systems, who found that $92 \%$ of systems belong to the thin disk (and are mostly young to middle age), $7.6 \%$ to the thick disk, and much less than $1 \%$ to the halo. Further inroads in this study can be made by collecting a larger volume-limited sample of very wide binaries and a comparison with a representative set of nearby field stars. We consider this paper as an initial step in this direction.

Despite the considerable progress in recent years, chronology of solar-type stars is still in a rather pitiful state, and we find more evidence of this in the widely discrepant age estimates for a few CPM systems obtained with different methods. Although a significant fraction of CPM companions display enhanced chromospheric, X-ray, and EUV activity, only few are patently in the pre-main-sequence stage of evolution (e.g., AT and AU Mic), where these signs of activity and the high rate of surface rotation can be attributed to a very young age. The origin of activity in most of our CPM systems lies in short-period binarity of their components, i.e., in hierarchical multiplicity. An interesting connection emerges between the presence of wide companions and the existence of short-period binaries. The reason for abundant multiple systems may partly be purely dynamical, in that the chances of survival are higher for systems with an internal binary because of the larger mass. An alternative astrophysical possibility is that the original fragmentation of a star-forming core takes place at various spatial scales and tends to produce multiple stellar systems, of which only hierarchical ones can survive for an appreciably long time.

Apparently, the timescale of dynamical survival of wide companions (of order $1 \mathrm{Gyr}$ ) is sufficiently long compared to the timescale of dynamical evolution of non-coplanar multiple systems (the Kozai cycle, $\S 7.1$ ) for the latter to shape up the present-day systems. The existence of circularized spectroscopic binaries with periods less than a few days may be the direct consequence of the interaction with remote companions, followed by the tidal friction and loss of angular momentum (Eggleton \& Kisseleva-Eggleton 2006). Ultimately, the inner components will form a contact binary and then merge. The existence of CPM multiple systems in a wide range of ages and separations will allow us to investigate this process in detail as it unfolds. Indeed, even in our sample of modest size we find examples of inner pairs of intermediate periods and large eccentricities, which are apparently evolving toward the tidally circularized state. The Kozai-type mechanism can affect the dynamical stability and composition of planetary systems. We find two stars in our sample with multiple planets $(55 \mathrm{Cnc}$ and GJ 777 A), and both have interesting dynamical properties very much unlike our solar system.

The research described in this paper was in part carried out at the Jet Propulsion Laboratory, California Institute of Technology, under a contract with the National Aeronautics and Space Administration (NASA). This research has made use of the SIMBAD database, operated at CDS, Strasbourg, France; and data products from the 2MASS, which is a joint project of the University of Massachusetts and the Infrared Processing and Analysis Center, California Technology Institute, funded by NASA and the National Science Foundation (NSF). 
Allen, C., \& Herrera, M. A. 1998, Rev. Mex. AA, 34, 37

Anosova, J. P., \& Orlov, V. V. 1991, A\&A, 252, 123

Balega, I. I., et al. 2006, A\&A, 448, 703

Barnes, S. A. 2007, ApJ, 669, 1167

Bartkevičius, A., \& Gudas, A. 2002, Baltic Astron., 11, 153

Bergeron, P., Legett, S. K., \& Ruiz, M. T. 2001, ApJS, 133, 413

Bessel, M. S. 1990, A\&AS, 83, 357

Butler, R. P., et al. 2006, ApJ, 646, 505

Chereul, E., et al. 1998, A\&A, 340, 384

Chiba, M., \& Beers, T. C. 2000, AJ, 119, 2843

Chmielewski, Y., et al. 1991, A\&A, 247, 368

Christian, D. J., et al. 2001, AJ, 122, 378

Cutispoto, G., et al. 2002, A\&A, 384, 491

Dahn, C. C., et al. 1982, AJ, 87, 419 2002, AJ, 124, 1170

del Peloso, E. F., da Silva, L., Porto de Mello, G. F. 2000, A\&A, 358, 233

Duquennoy, A., et al. 1992, A\&A, 254, 13

Eggen, O. J. 1993, AJ, 106, 1885 1995, AJ, 110, 1749 1998, AJ, 116, 284

Eggleton, P. P., \& Kisseleva-Eggleton, L. 2006, Ap\&SS, 304, 75

ESA. 1997, The Hipparcos and Tycho Catalogues (ESA SP-1200; Noordwijk: ESA)

Fabricius, C., \& Makarov, V. V. 2000a, A\&AS, 144, 45 2000b, A\&A, 356, 141

Famaey, B., et al. 2005, A\&A, 430, 165

Favata, F., Micela, G., \& Sciortino, S. 1997, A\&A, 322, 131

Frasca, A., Catalano, S., \& Mantovani, D. 1997, A\&A, 320, 101

Gizis, J. E., Reid, I. N., \& Hawley, S. H. 2002, AJ, 123, 3356

Gould, A., \& Chaname, J. 2004, ApJS, 150, 455

Gray, R. O., et al. 2003, AJ, 126, 2048 2006, AJ, 132, 161

Henry, T. J., et al. 2002, AJ, 123, 2002 2004, AJ, 128, 2460

Herbst, W., \& Miller, J. 1989, AJ, 97, 891

Hebb, L., et al. 2007, MNRAS, 379, 63

Høg E., et al. 2000, A\&A, 355, L27

Hünsch, M., et al. 1999, A\&AS, 135, 319

Innanen, K. A., et al. 1997, AJ, 113, 1915

Jancart, S., et al. 2005, A\&A, 442, 365

Kahanpää, J., et al. 1999, A\&A, 350, 513

Kiseleva, L. G., et al. 1998, MNRAS, 300, 292

Kiyaeva, O. V., et al. 2001, Astron. Lett., 27, 391

Koen, C., et al. 2002, MNRAS, 334, 20

Lépine, S., \& Bongiorno, B. 2007, AJ, 133, 889

Lépine, S., \& Shara, M. 2005, AJ, 130, 1483

Makarov, V. V. 2003, AJ, 126, 1996

- 2004, ApJ, 600, L71 2007a, ApJS, 169, 105 $2007 \mathrm{~b}$, ApJ, 658, 480

Makarov, V. V., \& Kaplan, G. H. 2005, AJ, 129, 2420

Makarov, V. V., Olling, R. P., \& Teuben, P. J. 2004, MNRAS, 352, 1199

Makarov, V. V., \& Urban, S. 2000, MNRAS, 317, 289

Makarov, V. V., et al. 2007, ApJ, 668, L155

Malkov, O. Y., et al. 2006, A\&A, 446, 785

Malmberg, D., Davies, M. B., \& Chambers, J. E. 2007, MNRAS, 377, L1

\section{REFERENCES}

Mason, B. D., et al. 2001, AJ, 122, 3466 2002, AJ, 124, 2254

Maxted, P. F. L., Marsh, T. R., \& Moran, C. K. J. 2000, MNRAS, 319, 305 Mazeh, T., et al. 2001, MNRAS, 325, 343

McArthur, B. E., et al. 2004, ApJ, 614, L81

Monet, D. G., et al. 2003, AJ, 125, 984

Montes, D., et al. 1997, A\&AS, 125, 263 2001, A\&A, 379, 976

Nidever, D. L., et al. 2002, ApJS, 141, 503

Nordström et al. 2004, A\&A, 418, 989

Osten, R. A., et al. 2000, ApJ, 544, 953

Patience, J., et al. 1998, AJ, 115, 1972

Phan-Bao, N., \& Bessell, M. S. 2006, A\&A, 446, 515

Poveda, A., et al. 1994, Rev. Mex. AA, 28, 43

Raghavan, D., et al. 2006, ApJ, 646, 523

Rauscher, E., \& Marcy, G. W. 2006, PASP, 118, 617

Reid, I. N., \& Cruz, K. L. 2002, AJ, 123, 2806

Reid, I. N., Hawley, S. L., Gizis, J. E. 1995, AJ, 110, 1838

Reid, I. N., Kilkenny, D., \& Cruz, K. L. 2002, AJ, 123, 2822

Reid, I. N., et al. 2003, AJ, 126, 3007

Reiners, A., \& Schmitt, J. H. M. M. 2003, A\&A, 398, 647

Roselló, G., et al. 1987, A\&AS, 67, 157

Rutten, R. G. M. 1986, A\&A, 159, 291

Salim, S., \& Gould, A. 2003, ApJ, 582, 1011

Scholz, A., et al. 2007, ApJ, 662, 1254

Seymour, D. M., et al. 2002, AJ, 123, 1023

Siess, L., Dufour, E., \& Forestini, M. 2000, A\&A, 358, 593

Silvestri, N. M., Oswalt, T. D., \& Hawley, S. L. 2002, AJ, 124, 1118

Soderblom, D. R., Duncan, D. K., \& Johnson, D. R. H. 1991, ApJ, 375, 722

Soubiran, C., \& Girard, P. 2005, A\&A, 438, 139

Stauffer, J. R., \& Hartmann, L. W. 1986, ApJS, 61, 531

Stern, R. A., Schmitt, J. H. M. M., \& Kahabka, P. T. 1995, ApJ, 448, 683

Takeda, Y., \& Kawanomoto, S. 2005, PASJ, 57, 45

Tokovinin, A., et al. 2006, A\&A, 450, 681

Tokovinin, A. A. 1990, Pis'ma Astron. Zh., 16, 52 1991, A\&AS, 91, 497

Torra, J., Fernández, D., \& Figueras, F. 2000, A\&A, 359, 82

Voges, W., et al. 1999, A\&A, 349, 389 2000, IAU Circ., 7432

Vogt, S. S., et al. 2005, ApJ, 632, 638

Weinberg, M. D., Shapiro, S. L., \& Wasserman, I. 1987, ApJ, 312, 367

Weis, E. W. 1988, AJ, 96, 1710 1991, AJ, 101, 1882

1993, AJ, 105, 1962 1996, AJ, 112, 2300

West, A. A., et al. 2006, AJ, 132, 2507 2008, AJ, 135, 785

Whitworth, A. P., \& Stamatellos, D. 2006, A\&A, 458, 817

Wright, J. T., et al. 2004, ApJS, 152, 261

Zacharias, N., McCallon, H. L., Kopan, E., \& Cutri, R. M. 2006, Extending the ICRF into the Infrared: 2MASS-UCAC Astrometry (26th IAU Meeting, Joint Discussion), 16

Zacharias, N. Urban, S. E., Zacharias, M. I., Wycoff, G. L., Hall, D. M., Monet, D. G., \& Rafferty, T. J. 2004a, AJ, 127, 3043

Zacharias, N., et al. 2004b, BAAS, 205, 4815 